\title{
Air Quality Impacts of Smallholder Oil Palm Processing in Nigeria
}

\author{
Elijah I. Ohimain ${ }^{1 *}$, Sylvester C. Izah ${ }^{1}$, Stephen O. Abah ${ }^{2}$ \\ ${ }^{1}$ Bioenergy and Environmental Biotechnology Research Unit, Department of Biological Sciences, Faculty of Science, Niger Delta \\ University, Wilberforce Island, Nigeria; ${ }^{2}$ Department of Community Health, Faculty of Clinical Sciences, College of Medicine, \\ Ambrose Alli University, Ekpoma, Nigeria. \\ Email: *eohimain@yahoo.com
}

Received May $18^{\text {th }}, 2013$; revised June $26^{\text {th }}, 2013$; accepted August $2^{\text {nd }}, 2013$

Copyright (C) 2013 Elijah I. Ohimain et al. This is an open access article distributed under the Creative Commons Attribution License, which permits unrestricted use, distribution, and reproduction in any medium, provided the original work is properly cited.

\begin{abstract}
Air emissions during palm oil processing by smallholders are issues of public health concern demanding urgent intervention by environmentalist. In this study, six smallholder oil palm processing mills were studied in Elele, Nigeria. Air emission parameters $\left(\mathrm{NO}_{2}, \mathrm{NH}_{3}, \mathrm{CO}, \mathrm{H}_{2} \mathrm{~S}, \mathrm{SO}_{2}, \mathrm{VOC}\right)$, noise and meteorology (wind speed, temperature, relative humidity and pressure) were determined at three distances (10 ft, $25 \mathrm{ft}$ and $50 \mathrm{ft}$ ) in both wind ward and lee ward directions from the mills covering boiling and digestion activities. The emissions from biomass were found to be significantly higher than that from fossil diesel, while noise was higher during digestion. The health implications of air emissions were discussed. The study concluded by directing attentions of regulatory agencies to monitor the activities of smallholder oil palm processing to ensure the environmental sustainability of their operations. In summary, evidence during boiling activity revealed that:

- $\mathrm{H}_{2} \mathrm{~S}$ ranged from $<0.01-2.400 \mathrm{ppm}$ at $10 \mathrm{ft},<0.01-2.067 \mathrm{ppm}$ at $25 \mathrm{ft}$ and $<0.01-0.833 \mathrm{ppm}$ at $50 \mathrm{ft}$ from the mills in the wind ward direction, and $<0.01-1.167 \mathrm{ppm}$ at $10 \mathrm{ft},<0.01-0.567 \mathrm{ppm}$ at $25 \mathrm{ft}$ and $<0.01-0.367 \mathrm{ppm}$ at $50 \mathrm{ft}$ distance from the mills in lee ward direction and was significantly lower during digestion.

- SPM ranged from $1634-7853 \mu \mathrm{g} / \mathrm{m}^{3}$ at $10 \mathrm{ft}, 657-1110 \mu \mathrm{g} / \mathrm{m}^{3}$ at $25 \mathrm{ft}$ and $81-854 \mu \mathrm{g} / \mathrm{m}^{3}$ at $50 \mathrm{ft}$ from the mills in the wind ward direction, and $46-236 \mu \mathrm{g} / \mathrm{m}^{3}$ at $10 \mathrm{ft}, 44-120 \mu \mathrm{g} / \mathrm{m}^{3}$ at $25 \mathrm{ft}$ and $30-58 \mu \mathrm{g} / \mathrm{m}^{3}$ at $50 \mathrm{ft}$ from the mills in lee ward direction. SPM was significantly lower during digestion.

- VOC ranged from $67-13.933 \mathrm{ppm}$ at $10 \mathrm{ft}, 1.033-13.133 \mathrm{ppm}$ at $25 \mathrm{ft}$ and $0.500-9.467 \mathrm{ppm}$ at $50 \mathrm{ft}$ from the mills in the wind ward direction, and $0.300-3.200 \mathrm{ppm}$ at $10 \mathrm{ft}, 0.133-6.733 \mathrm{ppm}$ at $25 \mathrm{ft}$ and $0.100-4.773 \mathrm{ppm}$ at $50 \mathrm{ft}$ from the mills in the lee ward direction, but was significantly lower during digestion..
\end{abstract}

Keywords: Air Emissions; Noise; Oil Palm Processing; Pollutant Gases

\section{Introduction}

Within the last few years, environmental issues are increasingly becoming relevant in economic activities and public health $[1,2]$ in Nigeria and the rest of the world. Of particular concern is the atmospheric environmental problems, which had previously received scanty attention in Nigeria but have become a subject of increasing National significance, particularly over the last two years [3].

Air pollution is a major threat to human life and most people inhale pollutants while at home or commuting to work irrespective of the mode of transportation [4]. De-

${ }^{*}$ Corresponding author. pending on the dose and the exposure time, these pollutant gases have the potential to cause far reaching adverse health effects in man, but principally affect the respiratory and cardiovascular systems. The World Health Organization (WHO) estimates that about 2.4 million people worldwide (including about 93,700 Nigerians) die each year from causes directly attributable to air pollution [5].

Oil palm (Elaeis guineensis) is an indigenous plant to West Africa [6]. It is the most productive oil crop in the world [7-15], accounting for 33\% of global vegetable oil production [16]. In Nigeria, over $80 \%$ of oil palm cultivation and production are controlled by smallholders [17, 18], who typically harvest semi wild plants and use 
manual processing techniques [19,20].

Unlike large oil palm processing mills, smallholders' operations are done manually and in batches, which depend on biomass fuel during boiling and diesel powered energy input during digestion (Figures 1 and 2). The increasing levels of oil palm cultivation and commercial processing in Nigeria thus raises serious environmental concerns due to air emissions generated during local oil palm production.

A hectare of oil palm produces 10 - 35 tonnes of fresh fruit bunch (FFB) per year [21-23]. The cultivation and processing of oil palm is a source of livelihood for many rural dwellers in Nigeria [18]. Oil palms are of multiple values and are crops of high economic importance that are often underscored [24]. The plantations cover a range of $1-5$ hectares of land and are characterized by mixed cropping to maximize the usage of the land [25].

There are at least eleven steps in the processing of oil palm fruits (Figure 1). In smallholder processing operations, all the steps are carried out manually without any external energy input except boiling and digestion. During oil palm processing activities, biomass such as Palm press fiber, empty fruit bunch, palm kernel shells and chaff are mainly used as boiler fuel, generating smoke.

Emissions are also generated from the diesel engine during digestion activity (Figure 2), releasing gaseous pollutants such as carbon monoxide (CO), sulphur dioxide $\left(\mathrm{SO}_{2}\right)$, nitrogen dioxide $\left(\mathrm{NO}_{2}\right)$, volatile organic compounds (VOCs) and particulate matter [26,27].

These emissions can be injurious to the environment [2] as well as humans [5,28-30]. $\mathrm{H}_{2} \mathrm{~S}$ and $\mathrm{NH}_{3}$ may also be emitted in minute quantities by the combustion of biomass and diesel fuel. The emission from diesel engines depends on the quality of the test fuels used $[31,32]$. Fossil diesel compositions include $40 \%$ paraffin, $35 \%$ of aromatic and $<10 \%$ of olefin [33]. $\mathrm{NO}_{2}$ is usually generated during combustion at high temperature [34], and its concentration increases with the engine combustion efficiency [35], while most $\mathrm{SO}_{2}$ from diesel engines is produced by the high-temperature oxidation of the sulfates

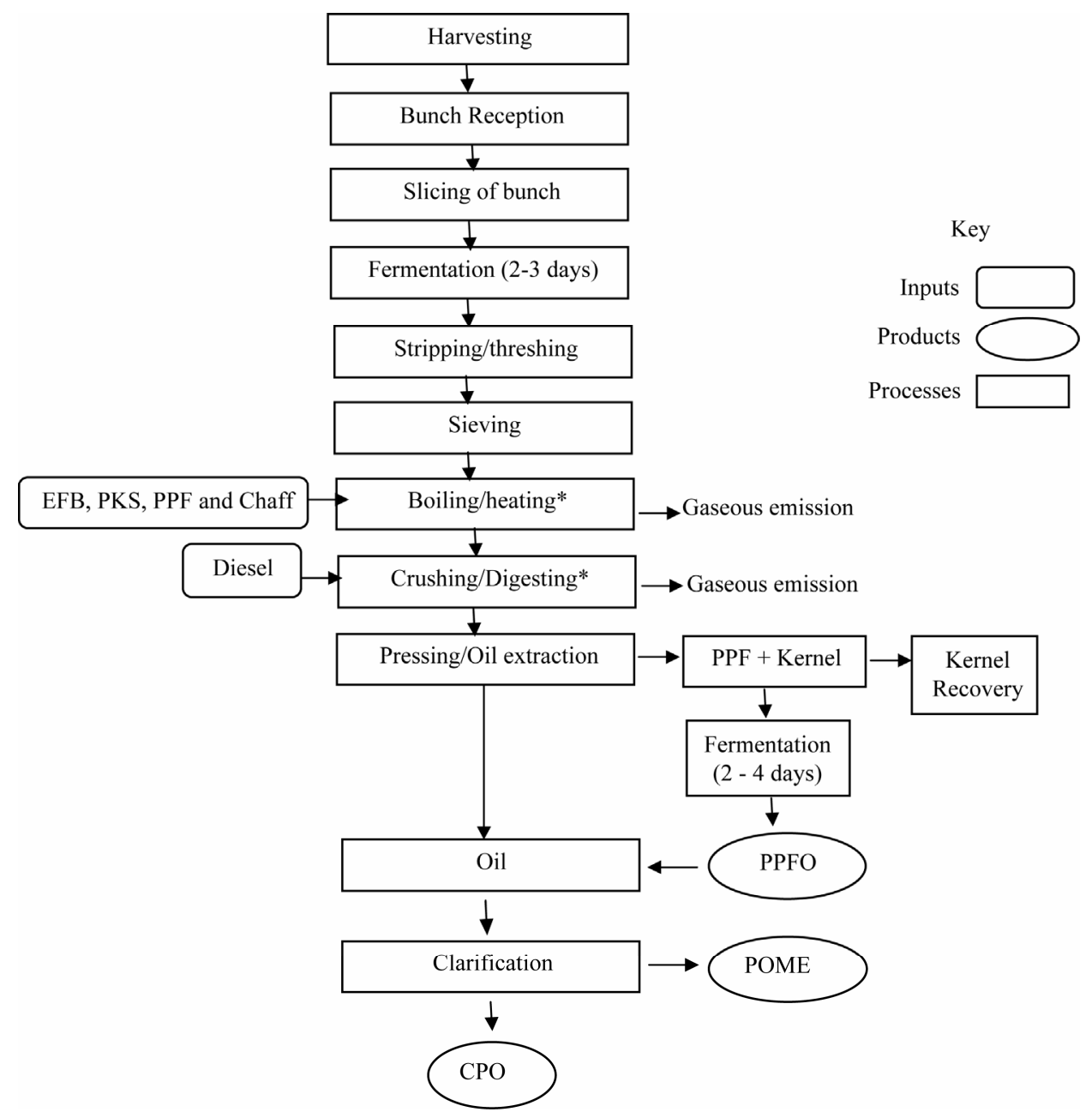

Figure 1. A schematic chart of palm oil processing by smallholders in Nigeria $(*=$ air quality sampling phase; POME $=$ palm oil milling effluents; $\mathrm{PPF}$ = palm press fibre; $\mathrm{PPFO}$ = palm press fibre oil; $\mathrm{CPO}$ = crude palm oil). 


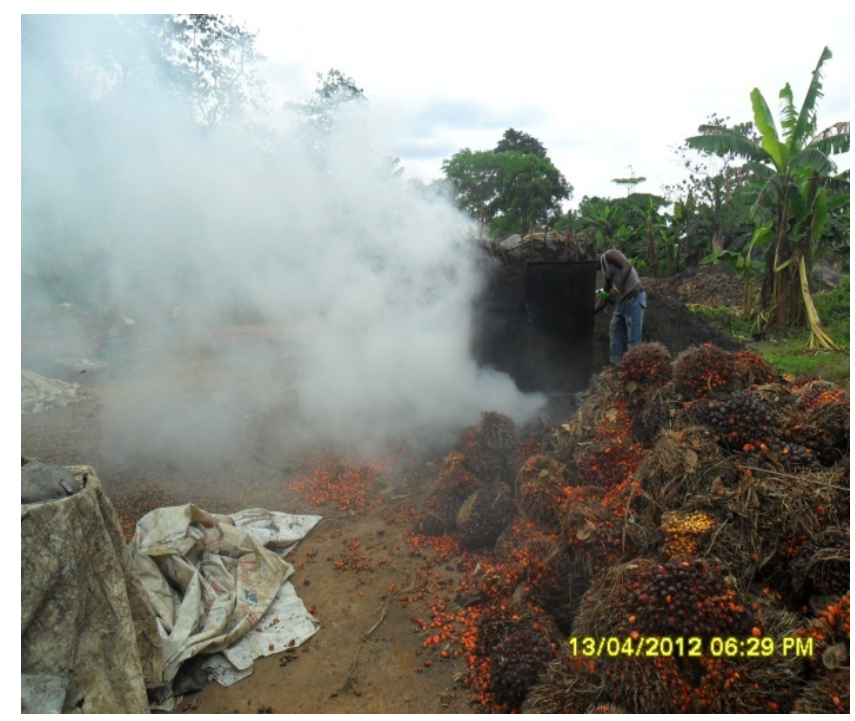

Boiling activity

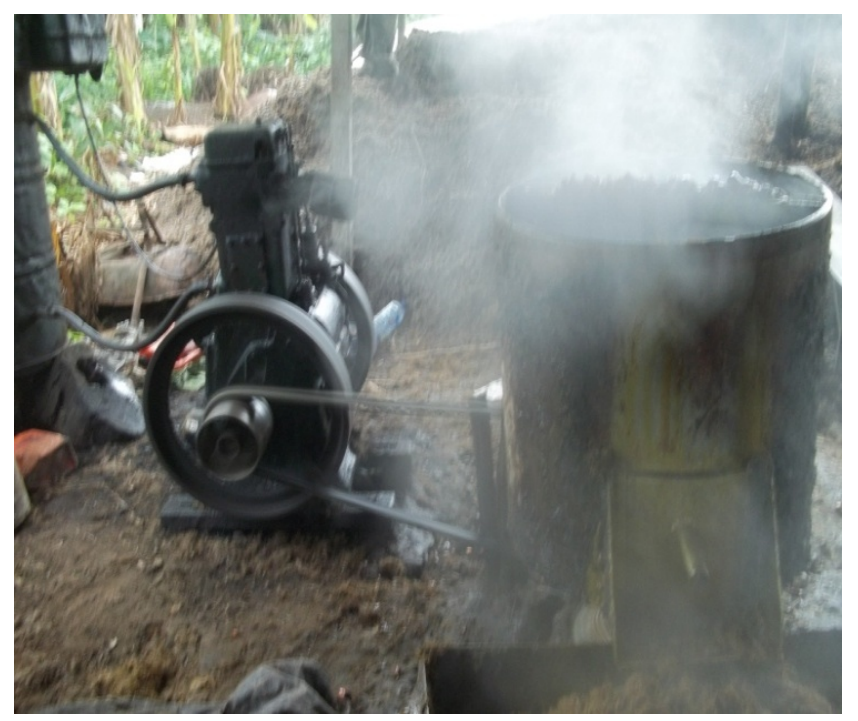

Digestion activity

Figure 2. Emission processes during oil palm processing by smallholders in Nigeria.

in petroleum diesel [34].

After they are emitted into the atmosphere, $\mathrm{NO}_{2}$ and $\mathrm{SO}_{2}$ become nitrates and sulfate aerosols respectively that detrimentally affect the environment. $\mathrm{SO}_{2}$ combined with water vapor in the atmosphere in the presence of a catalyst $\left(\mathrm{NO}_{2}\right)$ forms $\mathrm{H}_{2} \mathrm{SO}_{4}$, and causes acid rain. When these acids are consumed, they cause acidosis in human body, and excess accumulation in the human body can lead to death [36].

This underlies concerns over the environmental impact of the use of these fuels as power sources during palm oil processing by small holders. Emissions of smoke, hydrocarbon etc from burning of biomass (such as wood, crops) and fossil fuels (such as diesel engines and other transportation engines) have received much concern from the general public and environmentalists [28,37-44], and are one of the major environmental problems confronting Nigeria especially the Niger Delta Area, yet information regarding this is very scarce. Apart from data collected by a few individuals and corporate organizations at scattered locations, there is no comprehensive and empirical database on the magnitude of the hazard and its deleterious effects on the ecosystems and the people of the region $[30,45]$.

Noise is generated during all the phases of oil palm processing especially during digesting and oil extraction activity. The meteorological indicators (wind speed, relative humidity, temperature and pressure) of climate are important considerations during oil palm processing. Air humidity conditions, among other factors, may affect human comfort, and could lead to weather-related mortality $[46,47]$ and influences air pollution, which induces respiratory diseases [48]. Although Relative humidity $(\mathrm{RH})$ has an effect on the formation and size of secon- dary aerosols and therefore on the deposition, it is generally under investigated in urban climate research [49]. This may be related to the fact that humans have difficulties perceiving changes of the relative humidity [50] due to lack of sensory receptors for humidity [51].

In advanced oil palm producing countries, the sector is aware of the pollution associated with the processing activities and they are striving towards quality, environmental conservation through sustainable development and cleaner technology [52]. With the increasing uses of oil palm products, its production increases. So the sector must prepare for the potential challenges associated with it. Environmental pollution is a critical issue that requires attention on a dynamic basis despite the existence of environmental laws and regulations [53].

Air quality studies in Nigeria and particularly the Niger Delta area are still in its infant stage and faced with numerous challenges [30,45]. Apart from the issue of too few air pollution studies, a secondary problem is that they are often independently carried out, and government is not involved in systematic and consistent air quality assessment programmes as is being done in other parts of the world such as that carried out by the Environmental Protection Agency (EPA) in the United States [54]. Hence self regulatory environmental management tools like the ISO 1400 and life cycle assessment (LCA) have been adopted by the palm oil industries where systematic assessment checklists on the whole operation and unit processes and pollution prevention strategies could be effectively formulated and implemented in most advanced oil palm processing countries [52].

The self-regulatory approach by the sector will be essential to protect the environment. Improved air quality could serve as a single health promotion strategy that 
could be beneficial to all, since everybody commute and breathe air and air pollution is ubiquitous and widespread [4]. Therefore, it becomes pertinent to investigate the air emissions from smallholders' oil palm processing in Nigeria.

\section{Materials and Methods}

\subsection{Field Sampling}

Six (6) smallholder oil palm processing mill were visited in Elele, Rivers State, Nigeria from the 13th of April through the 22nd of April 2012. All the oil palm processing mills use similar processes and triplicate samples were collected from each mill specifically during boiling and digestion operations (Figures 1 and 2). The environmental components studied include: Air quality, Noise and meteorology.

\subsection{Air Quality/Meteorological Measurement}

The Air quality/meteorological parameters monitored include total Suspended Particulate, $\mathrm{CO}, \mathrm{SO}_{2}, \mathrm{NO}_{2}, \mathrm{NH}_{3}$, VOCs Noise, Wind speed, Atmospheric temperature, Pressure, and Relative humidity. Parameters such as $\mathrm{CO}$, $\mathrm{SO}_{2}, \mathrm{NO}_{2}, \mathrm{NH}_{3}, \mathrm{H}_{2} \mathrm{~S}$, VOCs and noise were measured using in-situ pre-calibrated portable air analyzers. Measurement was made at three (3) different distances from the mills $(10,25$ and $50 \mathrm{ft})$ in both the windward and lee-ward directions. The methodologies used for the air quality indicators are discussed below:

\subsubsection{Pollutant Gases}

Portable environmental air analyzers were employed for the air quality measurement of the pollutants and including: $\mathrm{NO}_{2}$ (Model number: $\mathrm{Z}-1400$ ), $\mathrm{SO}_{2}$ (Model number: $\mathrm{Z}-1300), \mathrm{NH}_{3}$ (Z-800), $\mathrm{H}_{2} \mathrm{~S}$ (Z-900), $\mathrm{CO}$ and VOCs (model ZDL-500). All the above equipment is product of Environmental Sensors Co, Boca Raton, Florida, USA) except VOC which is a product of met-one instrument, USA.

\subsubsection{Noise Level}

An Extech instrument (China), model 407730 Sound level meter with measuring range of $40-130 \mathrm{~dB}(\mathrm{~A})$, was used to measure the noise level at all the processing mills visited. Measurements were done by directing the probe towards the direction of the prevailing sound and the reading recorded from the digital meter in decibels dB.

\subsubsection{Suspended Particulate Matter (SPM)}

A Mini-volume air sampler (model: AEROCET 531, Manufactured by Met-one instrument, USA) with a preweighed membrane filter $(45 \mu \mathrm{m})$ was used to collect particulate matter. With the aid of a pump and a flowregulating device, air samples were pumped at a flow rate of 5 LPM at ambient conditions. Particle size separation was achieved by impaction and an impactor of 10micron cut-point was employed. A quartz filter of $47 \mathrm{~mm}$ diameter was used for trapping and a sensitive analytical microbalance was used for weighing.

\subsubsection{Meteorological Parameters}

The Kestrel (model: 4500 NV) manufactured by NielsenKellerman CO, Boothywn, USA meteorological station were used to measure temperature, relative humidity, wind speed and pressure.

\subsection{Statistical Analysis}

SPSS software version 17 (SPSS Inc., Chicago) was used to carry out the statistical analysis. A one-way analysis of variance was carried out at $\alpha=0.05$, and Duncan's multiple range test was used to discern the source of the observed differences.

\section{Results and Discussion}

The results of the air quality analysis during boiling and digestion of oil palm is presented in Tables 1 and $\mathbf{2}$ respectively, while noise/meteorology are respectively presented in Tables 3 and $\mathbf{4} . \mathrm{NO}_{2}$ concentration was generally below the equipment detection limits $(<0.01 \mathrm{ppm})$ in most of the mills, though with few exceptions.

During boiling, $\mathrm{NO}_{2}$ was recorded in few mills; at 10 $\mathrm{ft}$ from mill A $(0.233 \pm 0.033 \mathrm{ppm})$ and mill $\mathrm{D}(0.267 \pm$ $0.033 \mathrm{ppm})$ in the wind ward direction, At mill $\mathrm{E}, \mathrm{NO}_{2}$ was recorded at $25 \mathrm{ft}(0.133 \pm 0.033 \mathrm{ppm})$ in the wind ward direction and $10 \mathrm{ft}(1.167 \pm 0.033 \mathrm{ppm})$ in the lee ward direction (i.e. off wind direction). In mill $\mathrm{C}, \mathrm{NO}_{2}$ was not recorded in the wind ward direction, but only at $50 \mathrm{ft}(0.167 \pm 0.033 \mathrm{ppm})$ in the lee ward direction. The may be attributed to sudden change in wind direction as was observed in the particular mill during the study.

During digestion, $\mathrm{NO}_{2}$ was not recorded at any of the mills in the lee-ward direction but only in mill $\mathrm{A}, \mathrm{B}$ and $\mathrm{C}$ in the wind ward direction. In these mills, $\mathrm{NO}_{2}$ was consistently higher at the $10 \mathrm{ft}$ distance from the mills and least at $50 \mathrm{ft}$. Since the permissible limits of $\mathrm{NO}_{2}$ in Nigeria are $0.04-0.06 \mathrm{ppm}$ [55] (Table 5), it therefore follows that the limits were exceeded in some of the mills during boiling. However, the levels during the digestion process were within the Nigerian ambient air quality standards.

$\mathrm{NO}_{2}$ is acidic gas and an important indicator of air pollution as it correlates well with other air pollutant concentrations. $\mathrm{NO}_{2}$ is emitted during the combustion of fossil diesel and biomass and the variation in the concentration detected in this study is likely to be associated 
Table 1. Air quality during boiling activity of oil palm processing in the wind and lee ward directions.

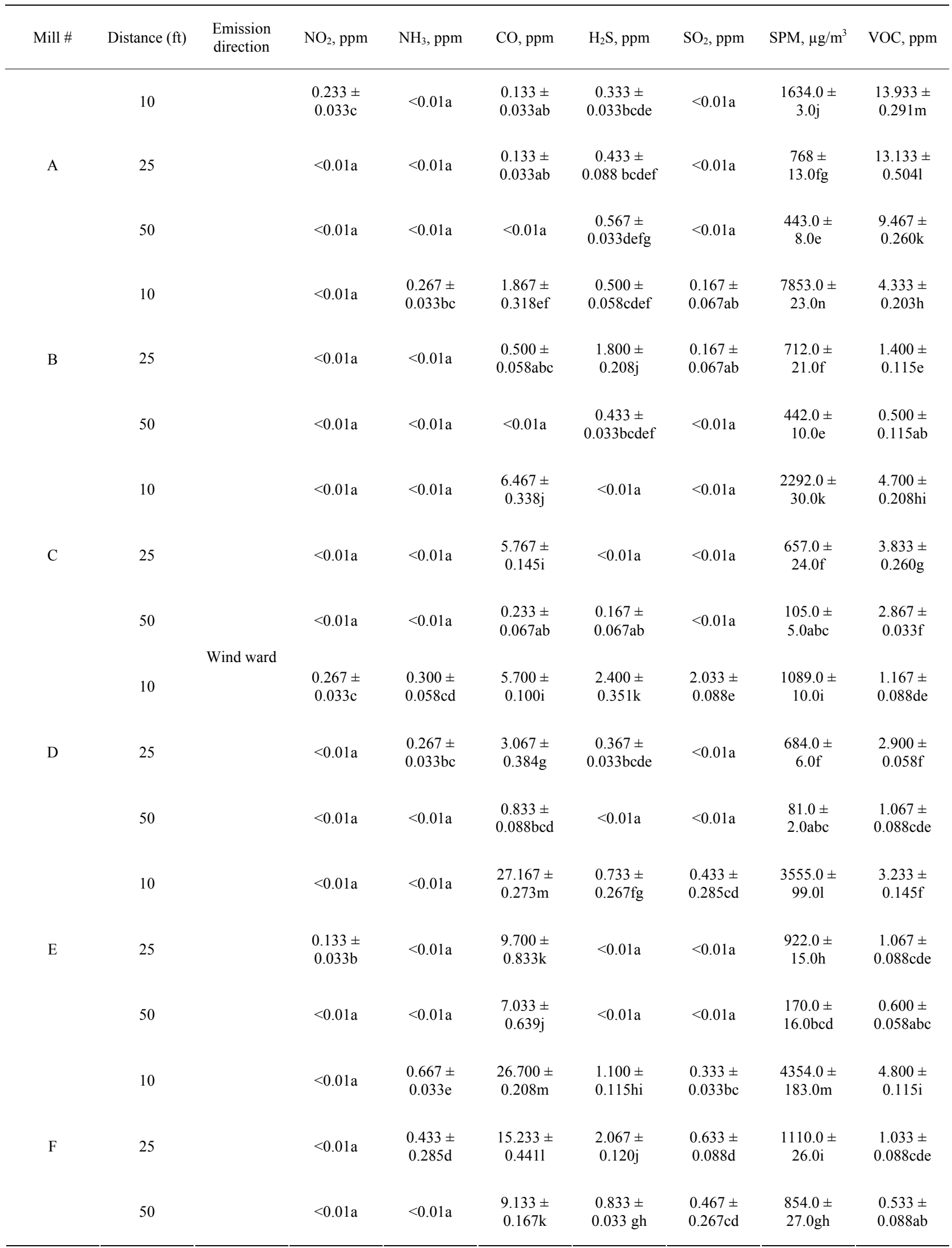




\section{Continued}

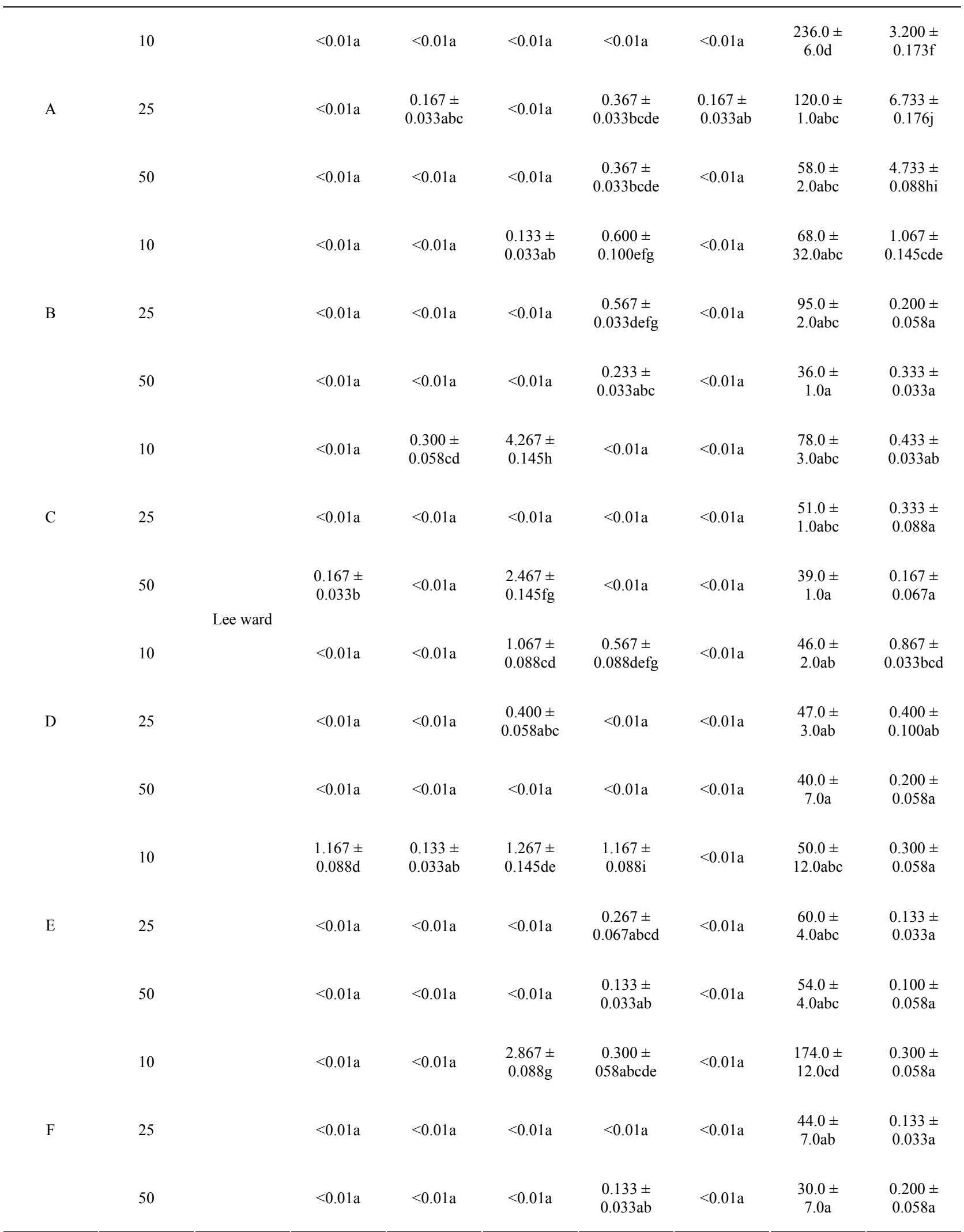

Each value is expressed as mean \pm standard error $(\mathrm{n}=3)$. Different letters in each column indicate significant differences at $P<0.05$ according to the Duncan Statistics. 
Table 2. Air quality during digestion activity of oil palm processing in the wind and lee ward directions.

\begin{tabular}{|c|c|c|c|c|c|c|c|c|c|}
\hline Mill \# & Distance (ft) & $\begin{array}{l}\text { Emission } \\
\text { direction }\end{array}$ & $\mathrm{NO}_{2}, \mathrm{ppm}$ & $\mathrm{NH}_{3}, \mathrm{ppm}$ & $\mathrm{CO}, \mathrm{ppm}$ & $\mathrm{H}_{2} \mathrm{~S}, \mathrm{ppm}$ & $\mathrm{SO}_{2}, \mathrm{ppm}$ & $\mathrm{SPM}, \mu \mathrm{g} / \mathrm{m}^{3}$ & VOC, ppm \\
\hline & 10 & & $\begin{array}{c}0.167 \pm \\
0.033 \mathrm{c}\end{array}$ & $\begin{array}{l}0.167 \pm \\
0.067 \mathrm{~d}\end{array}$ & $\begin{array}{l}0.700 \pm \\
0.115 \mathrm{hi}\end{array}$ & $\begin{array}{c}0.300 \pm \\
0.058 \mathrm{~b}\end{array}$ & $<0.01$ & $\begin{array}{l}163.0 \pm \\
2.01 \mathrm{pq}\end{array}$ & $\begin{array}{c}4.700 \pm \\
0.153 \mathrm{i}\end{array}$ \\
\hline \multirow[t]{3}{*}{ A } & 25 & & $\begin{array}{l}0.033 \pm \\
0.033 \mathrm{ab}\end{array}$ & $\begin{array}{l}0.067 \pm \\
0.033 \mathrm{bc}\end{array}$ & $\begin{array}{c}0.300 \pm \\
0.100 \text { abcde }\end{array}$ & $\begin{array}{c}0.033 \pm \\
0.033 \mathrm{a}\end{array}$ & $<0.01$ & $\begin{array}{c}30.0 \pm \\
6.0 \text { abcde }\end{array}$ & $\begin{array}{c}1.100 \pm \\
0.153 \mathrm{cde}\end{array}$ \\
\hline & 50 & & $\begin{array}{c}0.067 \pm \\
0.033 b\end{array}$ & $\begin{array}{l}0.033 \pm \\
0.033 \mathrm{ab}\end{array}$ & $\begin{array}{c}0.200 \pm \\
0.058 \mathrm{abcd}\end{array}$ & $\begin{array}{c}0.033 \pm \\
0.033 \mathrm{a}\end{array}$ & $<0.01$ & $\begin{array}{c}46.0 \pm \\
2.0 \text { defghi }\end{array}$ & $\begin{array}{l}0.433 \pm \\
0.067 \mathrm{ab}\end{array}$ \\
\hline & 10 & & $\begin{array}{c}0.133 \pm \\
0.033 \mathrm{c}\end{array}$ & $<0.01 \mathrm{a}$ & $\begin{array}{l}0.733 \pm \\
0.088 \mathrm{hi}\end{array}$ & $\begin{array}{c}0.033 \pm \\
0.033 \mathrm{a}\end{array}$ & $<0.01$ & $\begin{array}{c}57.0 \pm \\
\text { 3.0fghijk }\end{array}$ & $\begin{array}{l}8.633 \pm \\
0.219 \mathrm{~m}\end{array}$ \\
\hline \multirow[t]{3}{*}{ B } & 25 & & $\begin{array}{c}0.067 \pm \\
0.033 b\end{array}$ & $<0.01 \mathrm{a}$ & $\begin{array}{c}0.433 \pm \\
0.033 \mathrm{defg}\end{array}$ & $<0.01 \mathrm{a}$ & $<0.01$ & $\begin{array}{c}26.0 \pm \\
3.0 \mathrm{abcd}\end{array}$ & $\begin{array}{c}2.100 \pm \\
0.289 \mathrm{f}\end{array}$ \\
\hline & 50 & & $<0.01 \mathrm{a}$ & $<0.01 \mathrm{a}$ & $\begin{array}{c}0.167 \pm \\
0.067 \mathrm{abcd}\end{array}$ & $\begin{array}{c}0.033 \pm \\
0.033 \mathrm{a}\end{array}$ & $<0.01$ & $17.0 \pm 7.0 \mathrm{a}$ & $\begin{array}{l}0.500 \pm \\
0.115 \mathrm{ab}\end{array}$ \\
\hline & 10 & & $\begin{array}{c}0.233 \pm \\
0.067 \mathrm{~d}\end{array}$ & $<0.01 \mathrm{a}$ & $\begin{array}{l}0.733 \pm \\
0.088 \text { hi }\end{array}$ & $<0.01 \mathrm{a}$ & $<0.01$ & $\begin{array}{c}77.0 \pm \\
3.0 \mathrm{klmn}\end{array}$ & $\begin{array}{c}10.167 \pm \\
0.410 \mathrm{n}\end{array}$ \\
\hline \multirow[t]{3}{*}{$\mathrm{C}$} & 25 & & $\begin{array}{c}0.067 \pm \\
0.033 b\end{array}$ & $<0.01 \mathrm{a}$ & $\begin{array}{c}0.267 \pm \\
0.088 \text { abcde }\end{array}$ & $<0.01 \mathrm{a}$ & $<0.01$ & $\begin{array}{c}30.0 \pm \\
4.0 \mathrm{abcde}\end{array}$ & $\begin{array}{c}2.367 \pm \\
0.273 f\end{array}$ \\
\hline & 50 & & $<0.01 \mathrm{a}$ & $<0.01 \mathrm{a}$ & $\begin{array}{c}0.167 \pm \\
0.067\end{array}$ & $<0.01 \mathrm{a}$ & $<0.01$ & $\begin{array}{c}59.0 \pm \\
\text { 3.0fghijkl }\end{array}$ & $\begin{array}{l}0.533 \pm \\
0.067 \mathrm{ab}\end{array}$ \\
\hline & 10 & Wind ward & $<0.01 \mathrm{a}$ & $\begin{array}{c}0.100 \pm \\
0.058 \mathrm{c}\end{array}$ & $\begin{array}{l}0.700 \pm \\
0.100 \mathrm{hi}\end{array}$ & $<0.01 \mathrm{a}$ & $<0.01$ & $\begin{array}{c}117.0 \pm \\
3.0 \mathrm{o}\end{array}$ & $\begin{array}{l}5.767 \pm \\
0.285 \mathrm{k}\end{array}$ \\
\hline \multirow[t]{3}{*}{$\mathrm{D}$} & 25 & & $<0.01 \mathrm{a}$ & $\begin{array}{l}0.067 \pm \\
0.033 \mathrm{bc}\end{array}$ & $\begin{array}{c}0.333 \pm \\
0.088 \text { abcde }\end{array}$ & $\begin{array}{c}0.033 \pm \\
0.033 a\end{array}$ & $<0.01$ & $\begin{array}{l}84.0 \pm \\
6.0 \mathrm{mn}\end{array}$ & $\begin{array}{l}1.567 \pm \\
0.285 \mathrm{e}\end{array}$ \\
\hline & 50 & & $<0.01 \mathrm{a}$ & $<0.01 \mathrm{a}$ & $\begin{array}{c}0.267 \pm \\
0.088 \text { abcde }\end{array}$ & $<0.01 \mathrm{a}$ & $<0.01$ & $\begin{array}{c}70.0 \pm \\
4.0 \mathrm{ijklm}\end{array}$ & $\begin{array}{l}0.400 \pm \\
0.058 \mathrm{ab}\end{array}$ \\
\hline & 10 & & $<0.01 \mathrm{a}$ & $<0.01 \mathrm{a}$ & $\begin{array}{c}0.867 \pm \\
0.088 \mathrm{i}\end{array}$ & $<0.01 \mathrm{a}$ & $<0.01$ & $\begin{array}{c}78.0 \pm \\
6.0 \mathrm{klmn}\end{array}$ & $\begin{array}{c}6.233 \pm \\
0.1451\end{array}$ \\
\hline \multirow[t]{3}{*}{$\mathrm{E}$} & 25 & & $<0.01 \mathrm{a}$ & $<0.01 \mathrm{a}$ & $\begin{array}{c}0.600 \pm \\
0.058 \mathrm{fgh}\end{array}$ & $<0.01 \mathrm{a}$ & $<0.01$ & $\begin{array}{c}34.0 \pm \\
7.0 \mathrm{abcdef}\end{array}$ & $\begin{array}{l}1.267 \pm \\
0.273 \mathrm{de}\end{array}$ \\
\hline & 50 & & $<0.01 \mathrm{a}$ & $<0.01 \mathrm{a}$ & $\begin{array}{c}0.300 \pm \\
0.100 \mathrm{abcde}\end{array}$ & $\begin{array}{c}0.033 \pm \\
0.033 a\end{array}$ & $<0.01$ & $\begin{array}{c}18.0 \pm \\
1.0 \mathrm{ab}\end{array}$ & $\begin{array}{c}0.500 \pm \\
0.058 \mathrm{ab}\end{array}$ \\
\hline & 10 & & $<0.01 \mathrm{a}$ & $<0.01 \mathrm{a}$ & $\begin{array}{c}0.633 \pm \\
0.067 \mathrm{ghi}\end{array}$ & $<0.01 \mathrm{a}$ & $<0.01$ & $\begin{array}{c}68.0 \pm \\
9.0 \mathrm{hjklm}\end{array}$ & $\begin{array}{c}4.800 \pm \\
0.115 \mathrm{ij}\end{array}$ \\
\hline \multirow[t]{2}{*}{$\mathrm{F}$} & 25 & & $<0.01 \mathrm{a}$ & $<0.01 \mathrm{a}$ & $\begin{array}{c}0.333 \pm \\
0.088 \text { abcde }\end{array}$ & $\begin{array}{c}0.033 \pm \\
0.033 \mathrm{a}\end{array}$ & $<0.01$ & $\begin{array}{c}53.0 \pm \\
\text { 2.0efghijk }\end{array}$ & $\begin{array}{l}1.067 \pm \\
0.088 \mathrm{~cd}\end{array}$ \\
\hline & 50 & & $<0.01 \mathrm{a}$ & $<0.01 \mathrm{a}$ & $\begin{array}{c}0.133 \pm \\
0.033 \mathrm{abc}\end{array}$ & $<0.01 \mathrm{a}$ & $<0.01$ & $\begin{array}{l}20.0 \pm \\
5.0 \mathrm{abc}\end{array}$ & $\begin{array}{l}0.533 \pm \\
0.088 \mathrm{ab}\end{array}$ \\
\hline
\end{tabular}




\section{Continued}

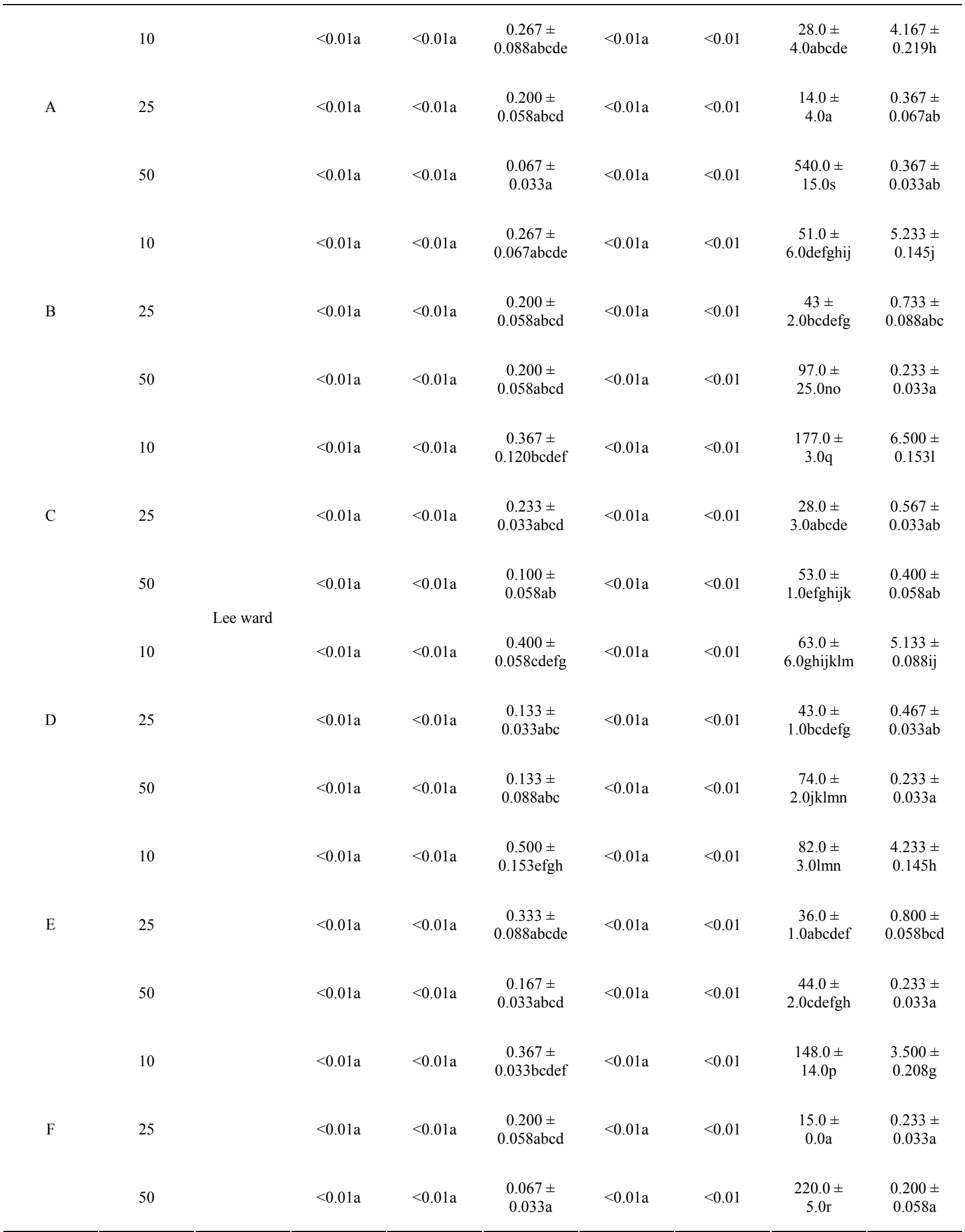

Each value is expressed as mean \pm standard error $(\mathrm{n}=3)$. Different letters in each column indicate significant differences at $P<0.05$ according to the Duncan Statistics. 
Table 3. Noise \& meteorology during boiling activity of oil palm processing in the wind and lee ward directions.

\begin{tabular}{|c|c|c|c|c|c|c|c|}
\hline Mill \# & $\begin{array}{l}\text { Distance } \\
\text { (ft) }\end{array}$ & $\begin{array}{l}\text { Emission } \\
\text { direction }\end{array}$ & Noise, $\mathrm{db}$ & Wind Speed, m/s & $\begin{array}{c}\text { Relative } \\
\text { Humidity RH, \% }\end{array}$ & Temperature, ${ }^{\circ} \mathrm{C}$ & Pressure, hpa \\
\hline & 10 & & $50.70 \pm 0.346 \mathrm{abc}$ & $0.400 \pm 0.058 \mathrm{bcdefgh}$ & $79.967 \pm 0.203 \mathrm{f}$ & $28.03 \pm 0.260$ abcde & $1008.50 \pm 0.058 \mathrm{efg}$ \\
\hline \multirow[t]{3}{*}{ A } & 25 & & $52.87 \pm 1.093 \mathrm{bc}$ & $0.267 \pm 0.033 \mathrm{abcde}$ & $84.900 \pm 0.346 \mathrm{kl}$ & $27.43 \pm 0.088 \mathrm{ab}$ & $1008.50 \pm 0.058 \mathrm{efg}$ \\
\hline & 50 & & $50.73 \pm 0.837 \mathrm{abc}$ & $0.167 \pm 0.067 \mathrm{ab}$ & $83.200 \pm 1.159 \mathrm{hij}$ & $27.53 \pm 0.033 \mathrm{abc}$ & $1008.43 \pm 0.033 \mathrm{efg}$ \\
\hline & 10 & & $68.90 \pm 0.416 \mathrm{f}$ & $0.133 \pm 0.033 \mathrm{ab}$ & $69.500 \pm 0.737 \mathrm{~cd}$ & $34.20 \pm 0.513 \mathrm{kl}$ & $1006.43 \pm 0.088 \mathrm{abc}$ \\
\hline \multirow[t]{3}{*}{ B } & 25 & & $63.50 \pm 0.300 \mathrm{e}$ & $0.233 \pm 0.067 \mathrm{abcd}$ & $68.367 \pm 0.145 c$ & $32.97 \pm 0.393 \mathrm{hi}$ & $1006.43 \pm 0.067 \mathrm{abc}$ \\
\hline & 50 & & $52.77 \pm 0.285 b c$ & $0.200 \pm 0.058 \mathrm{abc}$ & $70.233 \pm 0.233 d$ & $32.27 \pm 0.233 \mathrm{gh}$ & $1008.27 \pm 0.088 \mathrm{e}$ \\
\hline & 10 & & $52.70 \pm 0.265 b c$ & $0.533 \pm 0.033$ efghijk & $60.900 \pm 0.265 \mathrm{a}$ & $35.80 \pm 0.058 \mathrm{~m}$ & $1006.63 \pm 0.088 \mathrm{c}$ \\
\hline \multirow[t]{3}{*}{$\mathrm{C}$} & 25 & & $49.27 \pm 0.837 \mathrm{a}$ & $1.567 \pm 0.176 \mathrm{~m}$ & $62.433 \pm 0.260 \mathrm{a}$ & $35.40 \pm 0.173 \mathrm{~m}$ & $1006.63 \pm 0.088 \mathrm{c}$ \\
\hline & 50 & Wind & $61.77 \pm 0.338 \mathrm{de}$ & $0.233 \pm 0.067 \mathrm{abcd}$ & $65.600 \pm 0.643 b$ & $34.03 \pm 0.581 \mathrm{jkl}$ & $1007.27 \pm 0.186 \mathrm{~d}$ \\
\hline & 10 & & $62.93 \pm 0.546 \mathrm{e}$ & $0.767 \pm 0.033 \mathrm{jk}$ & $62.400 \pm 0.173 a$ & $34.57 \pm 0.2601$ & $1006.13 \pm 0.145 \mathrm{ab}$ \\
\hline \multirow[t]{3}{*}{$\mathrm{D}$} & 25 & & $49.53 \pm 0.617 \mathrm{ab}$ & $0.633 \pm 0.328$ ghijk & $64.533 \pm 0.176 b$ & $33.57 \pm 0.186 \mathrm{ijk}$ & $1006.07 \pm 0.088 \mathrm{a}$ \\
\hline & 50 & & $49.47 \pm 0.437 \mathrm{ab}$ & $0.733 \pm 0.033 \mathrm{ijk}$ & $64.500 \pm 0.265 b$ & $33.23 \pm 0.240 \mathrm{ij}$ & $1006.10 \pm 0.115 \mathrm{a}$ \\
\hline & 10 & & $62.43 \pm 0.788 \mathrm{de}$ & $0.800 \pm 0.058 \mathrm{k}$ & $81.800 \pm 0.451 \mathrm{gh}$ & $29.10 \pm 0.493 f$ & $1008.63 \pm 0.088 \mathrm{efg}$ \\
\hline \multirow[t]{3}{*}{$\mathrm{E}$} & 25 & & $61.30 \pm 0.493 \mathrm{de}$ & $0.800 \pm 0.058 \mathrm{k}$ & $83.667 \pm 1.081 \mathrm{ijk}$ & $28.50 \pm 0.173 \mathrm{def}$ & $1008.70 \pm 0.000 \mathrm{fg}$ \\
\hline & 50 & & $61.53 \pm 0.524 \mathrm{de}$ & $0.167 \pm 0.033 \mathrm{ab}$ & $85.167 \pm 0.769 \mathrm{klm}$ & $28.67 \pm 0.133 \mathrm{ef}$ & $1008.73 \pm 0.033 \mathrm{~g}$ \\
\hline & 10 & & $62.87 \pm 0.524 \mathrm{e}$ & $0.633 \pm 0.033$ ghijk & $73.033 \pm 0.674 \mathrm{e}$ & $27.93 \pm 0.145 \mathrm{abcde}$ & $1008.47 \pm 0.033 \mathrm{efg}$ \\
\hline \multirow[t]{3}{*}{$\mathrm{F}$} & 25 & & $61.87 \pm 1.037 \mathrm{de}$ & $0.700 \pm 0.058 \mathrm{ijk}$ & $86.467 \pm 0.7841 \mathrm{~m}$ & $28.30 \pm 0.153 \mathrm{cdef}$ & $1008.63 \pm 0.067 \mathrm{efg}$ \\
\hline & 50 & & $62.43 \pm 0.788 \mathrm{de}$ & $0.600 \pm 0.058$ ghijk & $72.500 \pm .351 \mathrm{e}$ & $27.73 \pm 0.088 \mathrm{abcd}$ & $1008.43 \pm 0.033 \mathrm{efg}$ \\
\hline & 10 & & $50.50 \pm 0.231 \mathrm{abc}$ & $0.467 \pm 0.033$ cdefghi & $80.300 \pm 0.153 \mathrm{fg}$ & $28.17 \pm 0.203$ bcde & $1008.33 \pm 0.186 \mathrm{efg}$ \\
\hline \multirow[t]{3}{*}{ A } & 25 & & $52.20 \pm 1.050 \mathrm{abc}$ & $0.133 \pm 0.033 \mathrm{ab}$ & $84.833 \pm 0.433 \mathrm{jkl}$ & $27.27 \pm 0.088 \mathrm{a}$ & $1008.43 \pm 0.176 \mathrm{efg}$ \\
\hline & 50 & & $50.73 \pm 0.736 \mathrm{abc}$ & $0.167 \pm 0.033 \mathrm{ab}$ & $83.033 \pm 1.087 \mathrm{hi}$ & $27.33 \pm 0.167 \mathrm{ab}$ & $1008.33 \pm 0.186 \mathrm{efg}$ \\
\hline & 10 & & $66.77 \pm 2.046 f$ & $0.567 \pm 0.067$ fghijk & $70.333 \pm 0.186 \mathrm{~d}$ & $33.90 \pm 0.458 \mathrm{jkl}$ & $1006.23 \pm 0.088 \mathrm{abc}$ \\
\hline \multirow[t]{3}{*}{ B } & 25 & & $62.50 \pm 0.755 \mathrm{de}$ & $0.300 \pm 0.058 \mathrm{abcdef}$ & $68.733 \pm 0.120 \mathrm{~cd}$ & $33.03 \pm 0.145 \mathrm{i}$ & $1006.27 \pm 0.088 \mathrm{abc}$ \\
\hline & 50 & & $53.17 \pm 0.433 c$ & $0.100 \pm 0.000 \mathrm{a}$ & $69.800 \pm 0.306 \mathrm{~cd}$ & $32.00 \pm 0.058 \mathrm{~g}$ & $1008.37 \pm 0.219 \mathrm{efg}$ \\
\hline & 10 & & $53.33 \pm 0.348 c$ & $0.633 \pm 0.120$ ghijk & $61.133 \pm 0.384 a$ & $35.63 \pm 0.120 \mathrm{~m}$ & $1006.53 \pm 0.067 b c$ \\
\hline \multirow[t]{3}{*}{$\mathrm{C}$} & 25 & & $50.43 \pm 0.696 a b c$ & $0.500 \pm 0.058$ defghij & $62.600 \pm 0.153 a$ & $35.50 \pm 0.173 \mathrm{~m}$ & $1006.53 \pm 0.120 b c$ \\
\hline & 50 & & $59.23 \pm 2.697 \mathrm{~d}$ & $0.267 \pm 0.088 \mathrm{abcde}$ & $65.66 \pm 0.6067 b$ & $33.93 \pm 0.533 \mathrm{jkl}$ & $1007.33 \pm 0.219 \mathrm{~d}$ \\
\hline & 10 & & $62.30 \pm 0.458 \mathrm{de}$ & $1.200 \pm 0.1151$ & $62.533 \pm 0.233 a$ & $34.60 \pm 0.2521$ & $1006.07 \pm 0.133 \mathrm{a}$ \\
\hline \multirow[t]{3}{*}{$\mathrm{D}$} & 25 & & $52.73 \pm 3.000 \mathrm{bc}$ & $1.367 \pm 0.0881 \mathrm{~m}$ & $64.467 \pm 0.145 b$ & $33.53 \pm 0.233 \mathrm{ijk}$ & $1006.10 \pm 0.153 \mathrm{a}$ \\
\hline & 50 & & $49.47 \pm 0.291 \mathrm{ab}$ & $0.667 \pm 0.088 \mathrm{hijk}$ & $64.433 \pm 0.145 b$ & $33.30 \pm 0.200 \mathrm{ij}$ & $1006.10 \pm 0.115 \mathrm{a}$ \\
\hline & 10 & & $63.43 \pm 1.235 \mathrm{e}$ & $0.667 \pm 0.088$ ghijk & $81.767 \pm 0.467 \mathrm{gh}$ & $29.07 \pm 0.088 \mathrm{f}$ & $1008.50 \pm 0.173 \mathrm{efg}$ \\
\hline \multirow[t]{3}{*}{ E } & 25 & & $62.87 \pm 1.450 \mathrm{e}$ & $0.567 \pm 0.067$ fghijk & $83.733 \pm 1.035 \mathrm{ijk}$ & $28.57 \pm 0.186 \mathrm{def}$ & $1008.57 \pm 0.145 \mathrm{efg}$ \\
\hline & 50 & & $60.90 \pm 1.153 \mathrm{de}$ & $0.367 \pm 0.067 \mathrm{abcdefg}$ & $85.133 \pm 0.745 \mathrm{klm}$ & $28.60 \pm 0.058 \mathrm{ef}$ & $1008.30 \pm 0.115 \mathrm{ef}$ \\
\hline & 10 & & $62.67 \pm 0.328 \mathrm{e}$ & $0.267 \pm 0.033 \mathrm{abcde}$ & $73.033 \pm 0.584 \mathrm{e}$ & $27.93 \pm 0.088 \mathrm{abcde}$ & $1008.60 \pm 0.173 \mathrm{efg}$ \\
\hline \multirow[t]{2}{*}{$\mathrm{F}$} & 25 & & $61.87 \pm 0.801 \mathrm{de}$ & $0.367 \pm 0.067 \mathrm{abcdefg}$ & $86.600 \pm 0.361 \mathrm{~m}$ & $28.40 \pm 0.153 \mathrm{def}$ & $1008.70 \pm 0.115 \mathrm{fg}$ \\
\hline & 50 & & $63.50 \pm 0.351 \mathrm{e}$ & $0.200 \pm 0.058 \mathrm{abc}$ & $72.2 \pm 0.20333 \mathrm{e}$ & $27.73 \pm 0.033 \mathrm{abcd}$ & $1008.40 \pm 0.153 \mathrm{efg}$ \\
\hline
\end{tabular}

Each value is expressed as mean \pm standard error $(\mathrm{n}=3)$. Different letters in each column indicate significant differences at $P<0.05$ according to the Duncan Statistics. 
Table 4. Noise \& meteorology during digestion activity of oil palm processing in wind and lee ward direction.

\begin{tabular}{|c|c|c|c|c|c|c|c|}
\hline Mill \# & $\begin{array}{c}\text { Distance } \\
(\mathrm{ft})\end{array}$ & $\begin{array}{l}\text { Emission } \\
\text { direction }\end{array}$ & Noise, $\mathrm{db}$ & Wind Speed, $\mathrm{m} / \mathrm{s}$ & $\mathrm{RH}, \%$ & Temperature, ${ }^{\circ} \mathrm{C}$ & Pressure, hpa \\
\hline \multirow{3}{*}{ A } & 10 & & $80.70 \pm 0.346 \mathrm{de}$ & $0.533 \pm 0.033 \mathrm{abcdefg}$ & $79.933 \pm 0.267 \mathrm{~h}$ & $28.03 \pm 0.291 \mathrm{abc}$ & $1008.67 \pm 0.088 \mathrm{ef}$ \\
\hline & 25 & & $74.47 \pm 0.145 \mathrm{c}$ & $0.267 \pm 0.033 \mathrm{abcd}$ & $84.733 \pm 0.285 \mathrm{kl}$ & $27.23 \pm 0.088 \mathrm{a}$ & $1008.37 \pm 0.120 \mathrm{ef}$ \\
\hline & 50 & & $64.07 \pm 2.652 \mathrm{a}$ & $0.167 \pm 0.067 \mathrm{ab}$ & $83.100 \pm 1.277 \mathrm{ijk}$ & $27.43 \pm 0.088 \mathrm{ab}$ & $1008.40 \pm 0.153 \mathrm{ef}$ \\
\hline \multirow{3}{*}{ B } & 10 & & $88.90 \pm 0.416 f$ & $0.200 \pm 0.058 \mathrm{abc}$ & $69.500 \pm 0.666 \mathrm{def}$ & $33.90 \pm 0.252 \mathrm{ghij}$ & $1006.43 \pm 0.145 \mathrm{abc}$ \\
\hline & 25 & & $73.50 \pm 0.300 \mathrm{c}$ & $0.167 \pm 0.067 \mathrm{ab}$ & $68.433 \pm 0.067 \mathrm{de}$ & $33.00 \pm 0.451 \mathrm{f}$ & $1006.20 \pm 0.058 \mathrm{abc}$ \\
\hline & 50 & & $66.10 \pm 3.508 \mathrm{a}$ & $0.200 \pm 0.058 \mathrm{abc}$ & $70.200 \pm 0.351 \mathrm{f}$ & $32.10 \pm 0.300 \mathrm{e}$ & $1008.17 \pm 0.088 \mathrm{e}$ \\
\hline \multirow{3}{*}{$\mathrm{C}$} & 10 & & $82.70 \pm 0.265 \mathrm{e}$ & $0.533 \pm 0.033$ abcdefg & $60.767 \pm 0.348 \mathrm{a}$ & $35.70 \pm 0.0581$ & $1006.57 \pm 0.033 \mathrm{~cd}$ \\
\hline & 25 & & $75.93 \pm 2.652 \mathrm{~cd}$ & $0.933 \pm 0.186 \mathrm{~g}$ & $62.533 \pm 0.088 b$ & $35.37 \pm 0.088 \mathrm{kl}$ & $1006.50 \pm 0.100 \mathrm{bc}$ \\
\hline & 50 & Wind & $63.10 \pm 1.002 \mathrm{a}$ & $0.567 \pm 0.067 \mathrm{abcdefg}$ & $65.500 \pm 0.529 c$ & $34.07 \pm 0.570 \mathrm{hij}$ & $1007.00 \pm 0.306 \mathrm{~d}$ \\
\hline \multirow{3}{*}{$\mathrm{D}$} & 10 & ward & $83.60 \pm 1.206 \mathrm{e}$ & $0.867 \pm 0.233 \mathrm{fg}$ & $62.467 \pm 0.088 b$ & $34.67 \pm 0.273 \mathrm{jk}$ & $1006.07 \pm 0.448 \mathrm{abc}$ \\
\hline & 25 & & $76.20 \pm 2.757 \mathrm{~cd}$ & $0.633 \pm 0.328 \mathrm{bcdefg}$ & $64.600 \pm 0.100 c$ & $33.57 \pm 0.088$ fghi & $1005.90 \pm 0.100 \mathrm{a}$ \\
\hline & 50 & & $66.80 \pm 2.425 \mathrm{ab}$ & $0.667 \pm 0.033 \mathrm{cdefg}$ & $64.400 \pm 0.058 c$ & $33.17 \pm 0.203 \mathrm{fg}$ & $1006.00 \pm 0.115 \mathrm{ab}$ \\
\hline \multirow{3}{*}{$\mathrm{E}$} & 10 & & $82.43 \pm 0.788 \mathrm{e}$ & $0.633 \pm 0.067 \mathrm{bcdefg}$ & $81.800 \pm 0.252 \mathrm{ij}$ & $29.03 \pm 0.481 \mathrm{~d}$ & $1008.37 \pm 0.176 \mathrm{ef}$ \\
\hline & 25 & & $74.63 \pm 2.948 \mathrm{c}$ & $0.800 \pm 0.058 \mathrm{efg}$ & $83.367 \pm 0.974 \mathrm{jkl}$ & $28.47 \pm 0.033 \mathrm{~cd}$ & $1008.50 \pm 0.153 \mathrm{ef}$ \\
\hline & 50 & & $64.53 \pm 1.369 \mathrm{a}$ & $0.600 \pm 0.200 \mathrm{abcdefg}$ & $84.967 \pm 0.9021 \mathrm{~m}$ & $28.57 \pm 0.033 \mathrm{~cd}$ & $1008.70 \pm 0.115 \mathrm{ef}$ \\
\hline \multirow{3}{*}{$\mathrm{F}$} & 10 & & $82.87 \pm 0.524 \mathrm{e}$ & $0.767 \pm 0.067 \mathrm{efg}$ & $73.100 \pm 0.709 \mathrm{~g}$ & $27.87 \pm 0.267 \mathrm{abc}$ & $1008.27 \pm 0.088 \mathrm{ef}$ \\
\hline & 25 & & $71.87 \pm 1.037 \mathrm{c}$ & $0.700 \pm 0.058 \mathrm{defg}$ & $86.467 \pm 0.612 \mathrm{~m}$ & $28.53 \pm 0.120 \mathrm{~cd}$ & $1008.60 \pm 0.115 \mathrm{ef}$ \\
\hline & 50 & & $64.43 \pm 1.178 \mathrm{a}$ & $0.733 \pm 0.088 \mathrm{defg}$ & $72.300 \pm 0.300 \mathrm{~g}$ & $27.40 \pm 0.058 \mathrm{ab}$ & $1008.27 \pm 0.088 \mathrm{ef}$ \\
\hline \multirow{3}{*}{ A } & 10 & & $80.57 \pm 0.418 \mathrm{de}$ & $0.533 \pm 0.033 \mathrm{abcdefg}$ & $79.900 \pm 0.361 \mathrm{~h}$ & $28.13 \pm 0.120 b c$ & $1008.73 \pm 0.033 f$ \\
\hline & 25 & & $74.20 \pm 0.058 \mathrm{c}$ & $0.267 \pm 0.033 \mathrm{abcd}$ & $84.500 \pm 0.351 \mathrm{kl}$ & $27.53 \pm 0.145 \mathrm{ab}$ & $1008.57 \pm 0.145 \mathrm{ef}$ \\
\hline & 50 & & $63.97 \pm 2.674 a$ & $0.133 \pm 0.033 a$ & $83.067 \pm 1.161 \mathrm{ijk}$ & $27.30 \pm 0.153 \mathrm{ab}$ & $1008.63 \pm 0.033 \mathrm{ef}$ \\
\hline \multirow{3}{*}{ B } & 10 & & $88.90 \pm 0.351 \mathrm{f}$ & $0.267 \pm 0.033$ & $69.267 \pm 0.498 \mathrm{def}$ & $34.03 \pm 0.348 \mathrm{hij}$ & $1006.43 \pm 0.145 a b c$ \\
\hline & 25 & & $73.60 \pm 0.252 \mathrm{c}$ & $0.333 \pm 0.145 \mathrm{abcd}$ & $68.200 \pm 0.058 \mathrm{~d}$ & $33.10 \pm 0.404 \mathrm{fg}$ & $1006.20 \pm 0.058 \mathrm{abc}$ \\
\hline & 50 & & $62.90 \pm 0.351 \mathrm{a}$ & $0.400 \pm 0.100 \mathrm{abcde}$ & $69.967 \pm 0.338 \mathrm{ef}$ & $31.90 \pm 0.252 \mathrm{e}$ & $1008.17 \pm 0.088 \mathrm{e}$ \\
\hline \multirow{3}{*}{$\mathrm{C}$} & 10 & & $82.53 \pm 0.285 \mathrm{e}$ & $0.533 \pm 0.033 \mathrm{abcdefg}$ & $60.667 \pm 0.318 \mathrm{a}$ & $35.40 \pm 0.100 \mathrm{kl}$ & $1006.30 \pm 0.058 \mathrm{abc}$ \\
\hline & 25 & & $75.57 \pm 2.696 \mathrm{~cd}$ & $0.933 \pm 0.186 \mathrm{~g}$ & $62.233 \pm 0.088 \mathrm{ab}$ & $35.60 \pm 0.0581$ & $1006.50 \pm 0.100 \mathrm{bc}$ \\
\hline & 50 & Lee & $63.33 \pm 0.984 a$ & $0.667 \pm 0.260 \mathrm{cdefg}$ & $65.267 \pm 0.521 \mathrm{c}$ & $34.30 \pm 0.557 \mathrm{ij}$ & $1006.30 \pm 0.058 \mathrm{abc}$ \\
\hline \multirow{3}{*}{$\mathrm{D}$} & 10 & ward & $83.53 \pm 1.284 \mathrm{e}$ & $0.700 \pm 0.100 \mathrm{defg}$ & $62.400 \pm 0.058 b$ & $34.67 \pm 0.273 \mathrm{jk}$ & $1006.07 \pm 0.448 \mathrm{abc}$ \\
\hline & 25 & & $75.90 \pm 2.762 \mathrm{~cd}$ & $0.700 \pm 0.265 \mathrm{defg}$ & $64.300 \pm 0.058 \mathrm{c}$ & $33.43 \pm 0.145 \mathrm{fgh}$ & $1006.23 \pm 0.120 \mathrm{abc}$ \\
\hline & 50 & & $66.67 \pm 2.153 \mathrm{ab}$ & $0.667 \pm 0.033 \mathrm{cdefg}$ & $64.200 \pm 0.058 c$ & $33.27 \pm 0.491 \mathrm{fgh}$ & $1006.17 \pm 0.088 \mathrm{abc}$ \\
\hline \multirow{3}{*}{$\mathrm{E}$} & 10 & & $82.33 \pm 0.636 \mathrm{e}$ & $0.700 \pm 0.265 \mathrm{defg}$ & $81.633 \pm 0.240 \mathrm{i}$ & $29.10 \pm 0.321 d$ & $1008.37 \pm 0.176 \mathrm{ef}$ \\
\hline & 25 & & $74.67 \pm 2.928 \mathrm{c}$ & $0.667 \pm 0.033 \mathrm{cdefg}$ & $83.267 \pm 1.017 \mathrm{ijkl}$ & $28.70 \pm 0.153 \mathrm{~cd}$ & $1008.50 \pm 0.153 \mathrm{ef}$ \\
\hline & 50 & & $64.33 \pm 1.485$ & $0.600 \pm 0.200 \mathrm{abcdefg}$ & $84.967 \pm 0.8651 \mathrm{~m}$ & $28.53 \pm 0.120 \mathrm{~cd}$ & $1008.70 \pm 0.115 \mathrm{ef}$ \\
\hline \multirow{3}{*}{$\mathrm{F}$} & 10 & & $82.50 \pm 0.300 \mathrm{e}$ & $0.667 \pm 0.033 \mathrm{cdefg}$ & $72.933 \pm 0.644 \mathrm{~g}$ & $27.90 \pm 0.252 \mathrm{abc}$ & $1008.37 \pm 0.145 \mathrm{ef}$ \\
\hline & 25 & & $71.60 \pm 0.945 b c$ & $0.400 \pm 0.208 \mathrm{abcdef}$ & $86.433 \pm 0.521 \mathrm{~m}$ & $28.60 \pm 0.173 \mathrm{~cd}$ & $1008.60 \pm 0.115 \mathrm{ef}$ \\
\hline & 50 & & $64.13 \pm 1.214 \mathrm{a}$ & $0.467 \pm 0.186 \mathrm{abcdefg}$ & $71.967 \pm 0.384 \mathrm{~g}$ & $27.17 \pm 0.088 \mathrm{a}$ & $1008.60 \pm 0.208 \mathrm{ef}$ \\
\hline
\end{tabular}

Each value is expressed as mean \pm standard error $(\mathrm{n}=3)$. Different letters in each column indicate significant differences at $P<0.05$ according to the Duncan Statistics. 
Table 5. Air quality, noise and meteorology recommendation guideline.

\begin{tabular}{|c|c|c|}
\hline \multirow{2}{*}{ Parameter } & \multicolumn{2}{|c|}{ NigerianAmbient Air Quality Standard [55] } \\
\hline & Time of average & Limits \\
\hline $\begin{array}{c}\text { Ambient } \\
\text { Temperature }{ }^{\circ} \mathrm{C}\end{array}$ & * & $30^{\circ} \mathrm{C}$ \\
\hline Noise $(\mathrm{dB}) \mathrm{A}$ & * & 90 \\
\hline $\mathrm{NO}_{2}(\mathrm{ppm})$ & $\begin{array}{c}\text { Daily average of hourly } \\
\text { values (range) }\end{array}$ & $\begin{array}{c}0.04 \mathrm{ppm}-0.06 \mathrm{ppm} \\
\left(75.0 \mu \mathrm{g} / \mathrm{m}^{3}-113 \mu \mathrm{g} / \mathrm{m}^{3}\right)\end{array}$ \\
\hline $\mathrm{NH}_{3}(\mathrm{ppm})$ & $*$ & $*$ \\
\hline $\mathrm{SO}_{2}(\mathrm{ppm})$ & $\begin{array}{c}\text { Daily average of hourly } \\
\text { values } 1 \text { hour }\end{array}$ & $\begin{array}{l}0.01 \mathrm{ppm}\left(26 \mu \mathrm{g} / \mathrm{m}^{3}\right) \\
0.1 \mathrm{ppm}\left(260 \mu \mathrm{g} / \mathrm{m}^{3}\right)\end{array}$ \\
\hline $\mathrm{H}_{2} \mathrm{~S}(\mathrm{ppm})$ & $*$ & $*$ \\
\hline $\mathrm{CO}(\mathrm{ppm})$ & $\begin{array}{l}\text { Daily average of hourly } \\
\text { values } 8 \text {-hourly average }\end{array}$ & $\begin{array}{l}10 \mathrm{ppm}\left(11.4 \mu \mathrm{g} / \mathrm{m}^{3}\right) \\
20 \mathrm{ppm}\left(22.8 \mu \mathrm{g} / \mathrm{m}^{3}\right)\end{array}$ \\
\hline VOC (ppm) & $*$ & $*$ \\
\hline $\operatorname{SPM}\left(\mu \mathrm{g} / \mathrm{m}^{3}\right)$ & $\begin{array}{c}\text { Daily average of hourly } \\
\text { values } 1 \text { hour }\end{array}$ & $\begin{array}{l}250 \mu \mathrm{g} / \mathrm{m}^{3} \\
600 \mu \mathrm{g} / \mathrm{m}^{3}\end{array}$ \\
\hline
\end{tabular}

"No stated limit.

with combustion temperature [56]. $\mathrm{NO}_{2}$ emission from digestion activity is dependent upon the speed of the engine and the load [57]. $\mathrm{NO}_{2}$ poses important environmental and public health concerns as it contributes to greenhouse gas levels and high levels of $\mathrm{NO}_{2}$ is associated with increased risk of respiratory diseases and contributes to heart, lung, liver and kidney diseases [30].

Ammonia was not generally detected in most of the mills during boiling and digestion activities, except in few instances. During boiling, ammonia was highest at $10 \mathrm{ft}(<0.01-0.667 \mathrm{ppm})$, followed by $25 \mathrm{ft}(<0.01-$ $0.267 \mathrm{ppm}$ ) and not detected at $50 \mathrm{ft}$ in the wind ward direction. Whereas in the lee ward direction ammonia was only recorded $(0.133-0.300 \mathrm{ppm})$ at $10 \mathrm{ft}$ distance from only 3 mills (A, C and E), and was not detected at other distances in the mills. Ammonia was generally absent during digestion both in the lee ward and wind ward directions except in mill $\mathrm{A}, \mathrm{B}, \mathrm{C}$ where it was recorded in the wind ward direction at $10 \mathrm{ft}(0.133-0.233 \mathrm{ppm}), 25$ $\mathrm{ft}(0.033-0.067 \mathrm{ppm})$ and $50 \mathrm{ft}(<0.01-0.067 \mathrm{ppm})$. Though, Nigeria does not have permissible limits for ammonia, the difference between the lee ward and wind ward concentration can be used to assess the impact. The result show that the concentration of ammonia released during boiling activity is significantly higher $(P<0.05)$ than that released during the digestion activity indicating that biomass fuel contribute higher levels of ammonia to the atmosphere than fossil fuel.

$\mathrm{CO}$ was detected at all the mills, during boiling and digestion activities at the 3 distances $(10 \mathrm{ft}, 25 \mathrm{ft}$ and 50 ft) from the mills in both wind ward and lee ward directions. During boiling, $\mathrm{CO}$ was $0.133-27.167 \mathrm{ppm}$ at 10 $10 \mathrm{ft}, 0.133-15.233 \mathrm{ppm}$ at $25 \mathrm{ft}$ and $<0.01-9.133 \mathrm{ppm}$ at $50 \mathrm{ft}$ in the wind ward direction, whereas in the lee ward direction it was significantly lower $(P<0.05)$ ranging from $<0.01-4.267 \mathrm{ppm}$ at $10 \mathrm{ft},<0.01-0.567$ ppm at $25 \mathrm{ft}$ and $<0.01-2.467 \mathrm{ppm}$ at $50 \mathrm{ft}$ from the mills. The higher emission generated at $25 \mathrm{ft}$ could be associated to the addition of more biomass/boiler fuel during measurement. During digestion, $\mathrm{CO}$ ranged from $0.633-0.867 \mathrm{ppm}$ at $10 \mathrm{ft}, 0.267-0.600 \mathrm{ppm}$ at $25 \mathrm{ft}$ and $0.133-0.300 \mathrm{ppm}$ at $50 \mathrm{ft}$ from the mils in the wind ward direction, whereas in the lee ward direction it ranged from $0.267-0.500 \mathrm{ppm}$ at $10 \mathrm{ft}, 0.133-0.333 \mathrm{ppm}$ at 25 $\mathrm{ft}$ and $0.067-0.200 \mathrm{ppm}$ at $50 \mathrm{ft}$ from the mills. $\mathrm{CO}$ was significantly higher during boiling than during digestion.

In all cases $\mathrm{CO}$ was highest in the $10 \mathrm{ft}$ distance and least at $50 \mathrm{ft}$ distance from the mills, the values recorded in the wind ward direction is significantly higher than those recorded in the lee ward direction. $\mathrm{CO}$ was higher than the Nigerian permissible limit of $10 \mathrm{ppm}$ (daily hourly average) and $20 \mathrm{ppm}$ (8-hourly average) [55] (Table 5) only during boiling activities thus indicating superiority of fossil fuel and higher combustion efficiency of the diesel generator compared to direct biomass burning. Typically, $\mathrm{CO}$ gas is produced by the incomplete combustion of carbonaceous materials or fossil fuels-gas, oil, coal and wood. The variation in the emission of $\mathrm{CO}$ is associated with the load, because the higher the load, the richer fuel mixture is burned, and thus produces more $\mathrm{CO}$ [56]. $\mathrm{CO}$ is of health concern as it inhibits the bloods ability to carry oxygen to vital organs such as the heart and brain.

The severity of the health effects is dose dependent. Typical sickness symptoms include headache, dizziness and nausea. $\mathrm{CO}$ is associated with reduced exercise tolerance, particularly in people with coronary artery disease, because of the formation of carboxyhaemoglobin [29,58].

During boiling activity, $\mathrm{H}_{2} \mathrm{~S}$ ranged from $<0.01-2.400$ ppm at $10 \mathrm{ft},<0.01-2.067 \mathrm{ppm}$ at $25 \mathrm{ft}$ and $<0.01-$ $0.833 \mathrm{ppm}$ at $50 \mathrm{ft}$ from the mills in the wind ward direction, whereas at the lee ward direction it was $<0.01$ $1.167 \mathrm{ppm}$ at $10 \mathrm{ft},<0.01-0.567 \mathrm{ppm}$ at $25 \mathrm{ft}$ and $<0.01$ - $0.367 \mathrm{ppm}$ at $50 \mathrm{ft}$ distance from the mills. During digestion activity, $\mathrm{H}_{2} \mathrm{~S}$ concentration ranged from $<0.01$ $0.300 \mathrm{ppm}$ at $10 \mathrm{ft},<0.01-0.033 \mathrm{ppm}$ at $25 \mathrm{ft}$ and $<0.01$ $-0.033 \mathrm{ppm}$ at $50 \mathrm{ft}$ in the wind ward direction, and was not detected in any of the mills in the lee ward direction.

Nigeria has no permissible limit for $\mathrm{H}_{2} \mathrm{~S}$, hence impact shall be established based on the differences between the values recorded in the wind ward direction relative to the lee ward direction. The pattern of variation in $\mathrm{H}_{2} \mathrm{~S}$ is similar to that observed in other air quality parameters, 
being highest during boiling activity than digestion and values recorded at the wind ward direction was significantly higher than the lee ward direction. In most of the mills, $\mathrm{H}_{2} \mathrm{~S}$ was lowest at $50 \mathrm{ft}$ from the mills i.e. the concentration decreases with increasing distance from the mills. These results also show the superiority of fossil fuel combustion over biomass in the processing of oil palm.

$\mathrm{SO}_{2}$ was recorded only in few instances during boiling activity only. During boiling, $\mathrm{SO}_{2}$ ranged from $<0.01-$ $2.033 \mathrm{ppm}$ at $10 \mathrm{ft},<0.01-0.633 \mathrm{ppm}$ at $25 \mathrm{ft}$ and $<0.01$ $-0.467 \mathrm{ppm}$ at $50 \mathrm{ft}$ from the mills in the wind ward direction, whereas at the lee ward direction, it was $<0.01$ ppm in all the mills except at $25 \mathrm{ft}$ from mill A where a value of $0.167 \pm 0.033 \mathrm{ppm}$ was recorded. $\mathrm{SO}_{2}$ was not detected at any distance during digestion activity. $\mathrm{SO}_{2}$ exceeded the permissible limits of $0.01-0.10 \mathrm{ppm}$ [55] (Table 5) during boiling activity, thus indicating the superiority of fossil fuel combustion over direct biomass combustion. Basically $\mathrm{SO}_{2}$ are produced by the combustion of fossil fuels containing sulphur.

SPM was very high especially during boiling activity ranging from $1634-7853 \mu \mathrm{g} / \mathrm{m}^{3}$ at $10 \mathrm{ft}, 657-1110$ $\mu \mathrm{g} / \mathrm{m}^{3}$ at $25 \mathrm{ft}$ and $81-854 \mu \mathrm{g} / \mathrm{m}^{3}$ at $50 \mathrm{ft}$ from the mills in the wind ward direction, whereas in the lee ward direction, it was $46-236 \mu \mathrm{g} / \mathrm{m}^{3}$ at $10 \mathrm{ft}, 44-120 \mu \mathrm{g} / \mathrm{m}^{3}$ at $25 \mathrm{ft}$ and $30-58 \mu \mathrm{g} / \mathrm{m}^{3}$ at $50 \mathrm{ft}$ from the mills.

During digestion, SPM ranged from $57-167 \mu \mathrm{g} / \mathrm{m}^{3}$ at $10 \mathrm{ft}, 14-84 \mu \mathrm{g} / \mathrm{m}^{3}$ at $25 \mathrm{ft}$ and $17-70 \mu \mathrm{g} / \mathrm{m}^{3}$ at $50 \mathrm{ft}$ from the mills in the wind ward direction, whereas in the lee ward direction it was $28-177 \mu \mathrm{g} / \mathrm{m}^{3}$ at $10 \mathrm{ft}, 14-43$ $\mu \mathrm{g} / \mathrm{m}^{3}$ at $25 \mathrm{ft}$ and $44-540 \mu \mathrm{g} / \mathrm{m}^{3}$ at $50 \mathrm{ft}$ from the mills. SPM was significantly higher $(P>0.05)$ during boiling activity than during digestion.

The highest value of SPM was recorded at $10 \mathrm{ft}$ distance to the mills in the wind ward direction, which decreased at increasing distance to the mills. The results also indicated that lower SPM was generated during digestion using diesel fossil fuel, again, showing that the diesel fossil fuel emit lesser SPM than wood fuels. While the SPM recorded during digestion activity were within the permissible limits of $250-600 \mu \mathrm{g} / \mathrm{m}^{3}$ [55] (Table 5) but SPM release during the boiling activity was significantly higher than this limit. The mass, size, number and particle composition are affected by combustion processes, condensation, adsorption, coagulation, agglomeration and collision of hydrocarbon in the engine exhaust $[59,60]$ Diesel exhaust (DE) is a major contributor to combustion derived particulate matter air pollution. The particulate matter generated is in the form of carbon black, soot and fly ash which are major components of smoke and are often within $10 \mu \mathrm{m}$ size range [45].

SPM exposure affects the lungs and heart, although the patho-physiological mechanism is not fully understood, due to the chemically heterogeneous nature of SPM [29]. Generally, however, it has been associated with decrease in lung function in children and adults, premature deaths from respiratory and cardiac causes and increased hospital admissions for asthma-like conditions [28]. Ossai et al. [61] and Efe [36] asserted that high rate of particulate matter causes respiratory diseases such as emphysema, pneumonia, bronchitis, asthma and respiratory tuberculosis. Also it can lead to eyes, teeth, and bones damage, increased susceptibility to disease and other stress-related environmental hazards, and reduces the reproduction potential in some species [62-65]. Other diseases associated with particulate matter include acute vascular dysfunction, increased thrombus formation pulmonary edema, etc. [45].

During boiling activity VOC ranged from 1.167 $13.933 \mathrm{ppm}$ at $10 \mathrm{ft}, 1.033-13.133 \mathrm{ppm}$ at $25 \mathrm{ft}$ and $0.500-9.467 \mathrm{ppm}$ at $50 \mathrm{ft}$ from the mills in the wind ward direction, while at the lee ward direction it was $0.300-3.200 \mathrm{ppm}$ at $10 \mathrm{ft}, 0.133-6.733 \mathrm{ppm}$ at $25 \mathrm{ft}$ and $0.100-4.773 \mathrm{ppm}$ at $50 \mathrm{ft}$ from the mills. During digestion, VOC ranged from $4.700-10.167 \mathrm{ppm}$ at $10 \mathrm{ft}$ and $1.067-2.367 \mathrm{ppm}$ at $25 \mathrm{ft}$ and $0.400-0.533 \mathrm{ppm}$ at $50 \mathrm{ft}$ from the mills in the wind ward direction, whereas in the lee ward direction, it was $3.500-6.500 \mathrm{ppm}$ at $10 \mathrm{ft}$, $0.233-0.800 \mathrm{ppm}$ at $25 \mathrm{ft}$ and $0.200-0.400 \mathrm{ppm}$ at $50 \mathrm{ft}$ from the mills. VOC was significantly higher during boiling activity than digestion, and in the wind ward than lee ward direction. The concentration of VOC was highest at $10 \mathrm{ft}$ to the mills and decreased with increasing distance to the mills. The low molecular weight organic fractions are highly volatile and with short atmospheric life-time.

Although the Nigerian ambient air quality standards does not specify any limit for VOC, health effects from these chemical compounds depend on the type, level and length of exposure. Short term exposure is likely cause of sensory irritation, particularly of the eyes, nose and throat [50] while long term exposure may lead to liver, kidney damage and cancer. However, VOCs could be affected byrelative humidity recorded in some mills $[66,67]$.

During boiling activity noise ranged from 50.70 $68.90 \mathrm{~dB}$ at $10 \mathrm{ft}, 49.27-63.50 \mathrm{~dB}$ at $25 \mathrm{ft}$ and $49.47-$ $62.43 \mathrm{~dB}$ at $50 \mathrm{ft}$ from the mills in the wind ward direction, whereas in the lee ward direction it was 50.50 $66.77 \mathrm{~dB}$ at $10 \mathrm{ft}, 50.43-62.87 \mathrm{~dB}$ at $25 \mathrm{ft}$ and 49.47 $63.50 \mathrm{~dB}$ at $50 \mathrm{ft}$ from the mills. During digestion, noise ranged from $80.70-88.90 \mathrm{~dB}$ at $10 \mathrm{ft}, 71.87-76.20 \mathrm{~dB}$ at $25 \mathrm{ft}$ and $63.10-66.80 \mathrm{~dB}$ at $50 \mathrm{ft}$ from the mills in the wind ward direction, whereas in the lee ward direction, it was $80.57-88.90 \mathrm{~dB}$ at $10 \mathrm{ft}, 71.60-75.90 \mathrm{~dB}$ at $25 \mathrm{ft}$ and $62.90-66.67 \mathrm{~dB}$ at $50 \mathrm{ft}$ from the mills.

There were no significant difference $(P>0.05)$ in the noise levels recorded at the wind ward and lee ward di- 
rection, indicating that the intensity of noise is unaffected by wind direction. From these results it will appear that noise pollution is not of serious concern during local processing of palm oil. Unlike in the other parameters, noise do not follow wind direction and was significantly higher $(P<0.05)$ during digestion than during boiling. This is expected because the Lister engine generates noise during digestion. Noise level was below the FEPA stipulated limit of $90 \mathrm{~dB}$ [55] (Table 5) during boiling and digestion activities.

During boiling activity, atmospheric temperature ranged from $27.93^{\circ} \mathrm{C}-35.80^{\circ} \mathrm{C}$ at $10 \mathrm{ft}, 27.43^{\circ} \mathrm{C}-$ $35.40^{\circ} \mathrm{C}$ at $25 \mathrm{ft}$ and $27.53^{\circ} \mathrm{C}-34.03^{\circ} \mathrm{C}$ at $50 \mathrm{ft}$ from the mills in the wind ward direction, whereas it was $27.93^{\circ} \mathrm{C}$ $-35.63^{\circ} \mathrm{C}$ at $10 \mathrm{ft}, 27.27^{\circ} \mathrm{C}-35.50^{\circ} \mathrm{C}$ at $25 \mathrm{ft}$ and $27.33^{\circ} \mathrm{C}$ $-35.93^{\circ} \mathrm{C}$ at $50 \mathrm{ft}$ from the mills in the lee ward direction. During digestion, temperature ranged from $27.87^{\circ} \mathrm{C}$ $35.70^{\circ} \mathrm{C}$ at $10 \mathrm{ft}, 27.23^{\circ} \mathrm{C}-35.37^{\circ} \mathrm{C}$ at $25 \mathrm{ft}$ and $27.40^{\circ} \mathrm{C}-$ $34.07^{\circ} \mathrm{C}$ at $50 \mathrm{ft}$ to the mills in the wind ward direction, whereas in the lee ward direction it was $27.93^{\circ} \mathrm{C}$ $35.40^{\circ} \mathrm{C}$ at $10 \mathrm{ft}, 27.53^{\circ} \mathrm{C}-35.60^{\circ} \mathrm{C}$ at $25 \mathrm{ft}$ and $27.17^{\circ} \mathrm{C}-$ $34.30^{\circ} \mathrm{C}$ at $50 \mathrm{ft}$ from the mills. The temperature was found to be greater that the permissible limit of $30^{\circ} \mathrm{C}$ [55] (Table 5). Though temperature decreased at increasing distances from the mills, there were no significant differences between the values recorded at the wind ward and lee ward wind directions and between boiling and digestion processing activities.

The wind speed ranged from $0.100-1.567 \mathrm{~m} / \mathrm{s}$ during boiling and $0.133-0.933 \mathrm{~m} / \mathrm{s}$ during digestion, which is classified as light air in the Beaufort scale. The wind speed and direction affected the concentration of air quality parameters but did not influence noise and temperature significantly. Relative humidity ranged from $62.40 \%$ - $86.60 \%$ during boiling and $60.79 \%-86.43 \%$ during digestion, while pressure ranged from 1006.07 1008.73 hpa during boiling and 1006.00 - $1008.87 \mathrm{hpa}$ during digestion. It does appear that relative humidity and pressure has no differential effects on the air quality parameters. The high values obtained in some mills and the distance from the processing activity may be attributed to reduced cloud cover and the influence of moisture laden tropical maritime air mass. However, RH appears to enhance particle deposition $[67,68]$.

The occasional increase in the emission from the longer distance as compared to short distance in some of the parameters is associated to the fueling of boiler fuel during measurement while the digestion activity was enhanced by the loading rate. Other factors are changes in wind speed and direction. This basically influenced both direction (lee and wind ward) measurements.

\section{Conclusion}

This study investigated the air quality during palm oil processing by smallholders in Nigeria. The air quality parameters $\left(\mathrm{NO}_{2}, \mathrm{NH}_{3}, \mathrm{CO}, \mathrm{H}_{2} \mathrm{~S}, \mathrm{SO}_{2}, \mathrm{VOC}\right.$, SPM) during boiling activity were found to exceed the threshold limits. Emissions during digestion activity were however within the threshold limits. Therefore, emissions during the boiling process could pose serious environmental and public health concern which portends negative implications for environmental sustainability. We recommend the use of improved burners that could emit fewer pollutants. Also, government should develop environmental quality guidelines for smallholder oil palm processing activities to ensure environmental sustainability of their operations.

\section{Acknowledgements}

The authors gratefully acknowledges the following undergraduates students of Niger Delta University that participated in the field sampling exercise; Eke Rita, Inatimi Sampson, Obieze Francis, Jeremiah Ikakita, Okonkwo Amaka, Oghenegueke Ejiroghene, Alaka Evelyn, Ereke Samuel, Kakiri Gift, Perewarebo Glory, Biriduba Woyengitonyokopa, Clarke Ebisine and Eretinghe Dorcas. The authors also wish to thank Ally Bedford of IDC Ltd. for reviewing the manuscript.

\section{REFERENCES}

[1] S. O. Abah, "HIA Practices in Nigeria," Impact Assessment and Project Appraisal, Vol. 30, No. 3, 2012, pp. 207-213. doi:10.1080/14615517.2012.705064

[2] E. I. Ohimain, E. I. Seiyaboh, S. C. Izah, V. E. Oghenegueke and T. G. Perewarebo, "Some Selected PhysicoChemical and Heavy Metal Properties of Palm Oil Mill Effluents," Greener Journal of Physical Sciences, Vol. 2, No. 4, 2012, pp. 131-137.

[3] T. F. Ediagbonya, E. E. Ukpebor, F. E. Okieimen and G. E. Okungbowa, "Comparative Study of TSP, Inhalable Particles and Respirable Particles in Urban and Rural Areas in Niger Delta Region of Nigeria," Greener Journal of Physical Sciences, Vol. 2, No. 3, 2012, pp. 89-96.

[4] C. E. Ekpenyong, E. O. Ettebong, E. E. Akpan, T. K. Samson and N. E. Daniel, "Urban City Transportation Mode and Respiratory Health Effect of Air Pollution: A Cross-Sectional Study among Transit and Non-Transit Workers in Nigeria," BMJ Open, Vol. 2, No. 5, 2012, Article ID: e001253. doi:10.1136/bmjopen-2012-001253

[5] World Health Organization, "Estimated Deaths and DALYs Attributable to Selected Environmental Risk Factors by WHO Member State, 2002," 2012.

http://www.who.int/entity/quantifying_ehimpacts/country profilesebd.xls

[6] S. O. Aghalino, "British Colonial Policies and the Oil Palm Industry in the Niger Delta Region of Nigeria 1900-1960," African Study Monographs, Vol. 21, No. 1, 2000, pp. 19-33.

[7] J. A. Akangbe, G. B. Adesiji, S. B. Fakayode and Y. O. 
Aderibigbe, "Towards Palm Oil Self-Sufficiency in Nigeria: Constraints and Training Needs Nexus of Palm Oil Extractors," Journal of Human Ecology, Vol. 33, No. 2, 2011, pp. 139-145.

[8] S. M. A. Tagoe, M. J. Dickinson and M. M. Apetorgbor, "Factors Influencing Quality of Palm Oil Produced at the Cottage Industry Level in Ghana," International Food Research Journal, Vol. 19, No. 1, 2012, pp. 271-278.

[9] J. N. Okechalu, M. M. Dashen, P. M. Lar, B. Okechalu and T. Gushop, "Microbiological Quality and Chemical Characteristics of Palm Oil Sold within Jos Metropolis, Plateau State, Nigeria," Journal of Microbiology and Biotechnology Research Vol. 1, No. 2, 2011, pp. 107-112.

[10] S. C. Izah and E. I. Ohimain, "Microbiological Quality of Crude Palm Oil Produced by Smallholder Processors in the Niger Delta, Nigeria," Journal of Microbiology and Biotechnology Research, Vol. 3, No. 2, 2013, pp. 30-36.

[11] E. G. F. Ngando, M. E. A. Mpondo, E. E. L. Dikotto and P. Koona, "Assessment of the Quality of Crude Palm Oil from Smallholders in Cameroon," Journal of Stored Products and Postharvest Research, Vol. 2, No. 3, 2011, pp. 52-58.

[12] M. U. Dimelu and V. Anyaiwe, "Priorities of Smallholder Oil Palm Producers in Ika Local Government Area of Delta State: Implication for Agricultural Extension Service in Nigeria," World Journal of Agricultural Sciences, Vol. 7, No. 2, 2011, pp. 117-123.

[13] P. F. Rupani, R. P. Singh, H. Ibrahim and N. Esa, "Review of Current Palm Oil Mill Effluent (POME) Treatment Methods: Vermicomposting as a Sustainable Practice," World Applied Sciences Journal, Vol. 11, No. 1, 2010, pp. 70-81.

[14] S. Sumathi, S. P. Chai and A. R. Mohamed, "Utilization of Oil Palm as a Source of Renewable Energy in Malaysia," Renewable and Sustainable Energy Review, Vol. 12, No. 9, 2008, pp. 2404-2421. doi:10.1016/j.rser.2007.06.006

[15] M. M. Ugbah and C. N. Nwawe, "Trends in Oil Palm Production in Nigeria," Journal of Food, Agriculture and Environment, Vol. 6, No. 1, 2008, pp. 119-122.

[16] United State Department of Agriculture (USDA), "World Agricultural Production," Foreign Agriculture Series WAP 11-12, USDA, Washington DC, 2012.

[17] S. Vermeulen and N. Guad, "Towards Better Practice in Small Holder Palm Oil Production," National Resources Issue Series No. 5, International Institute for Environment and Development, London, 2006.

[18] E. I. Ohimain, C. Daokoru-Olukole, S. C. Izah and E. E. Alaka, "Assessment of the Quality of Crude Palm Oil Produced by Smallholder Processors in Rivers State, Nigeria," Nigerian Journal of Agriculture, Food and Environment, Vol. 8, No. 2, 2012, pp. 28-34.

[19] E. I. Ohimain, A. A. Oyedeji and S. C. Izah, "Employment Effects of Smallholder Oil Palm Processing Plants in Elele, Rivers State, Nigeria," International Journal of Applied Research and Technology, Vol. 1, No. 6, pp. 8393.

[20] F. I. Olagunju, "Economics of Palm Oil Processing in
Southwestern Nigeria," International Journal of Agricultural Economics and Rural Development, Vol. 1, No. 2, 2008, pp. 69-77.

[21] M. K. C. Sridhar and O. O. AdeOluwa, "Palm Oil Industry Residue," In: P. S. Nigam and A. Pandey, Eds., Biotechnology for Agro-Industrial Residues Utilization, Springer Science, Berlin, 2009, pp. 341-355.

[22] R. P. Singh, A. Embrandiri, M. H. Ibrahim and N. Esa, "Management of Biomass Residues Generated from Oil Mill: Vermicomposting a Sustainable Option," Resources, Conservation and Recycling, Vol. 55, No. 4, 2011, pp. 423-434. doi:10.1016/j.resconrec.2010.11.005

[23] R. P. Singh, M. H. Ibrahim and N. Esa, "Composting of Waste from Palm Oil Mill: A Sustainable Waste Management Practice," Review in Environmental Science Biotechnology, Vol. 9, No. 4, 2010, pp. 331-344. doi:10.1007/s11157-010-9199-2

[24] Foundation for Partnership Initiatives in the Niger Delta (PIND), "A report on Palm Oil Value Chain Analysis in the Niger Delta," 2011, pp. 2-4.

www.pindfoundation.net/wp-content/plugins/.../download .php?id=28

[25] Initiative for Public Policy Analysis (IPPA), "African Case Study: Palm Oil and Economic Development in Nigeria and Ghana; Recommendations for the World Bank's 2010 Palm Oil Strategy," 2010. http://www.ippanigeria.org/press/2.html

[26] I. Colbeck, Z. A. Nasir, S. Ahmad and Z. Ali, "Exposure to PM10, PM2.5, PM1 and Carbon Monoxide on Roads in Lahore, Pakistan," Aerosol and Air Quality Research, Vol. 11, 2001, pp. 689-695.

[27] C. M. Ma, G. B. Hong and C. T. Chang, "Influence of Traffic Flow Patterns on Air Quality inside the Longest Tunnel in Asia," Aerosol and Air Quality Research, Vol. 11, 2011, pp. 44-50.

[28] T. Adler, "Respiratory Health: Measuring the Health Effects of Crop Burning," Environ Health Perspectives, Vol. 118, No. 11, 2010, p. A475. doi:10.1289/ehp.118-a475

[29] A. Abelsohn, D. Stieb, M. D. Sanborn and E. Weir, "Identifying and Managing Adverse Environmental Health Effects: 2. Outdoor Air Pollution," CMAJ, Vol. 166, No. 9, 2002, pp. 161-167.

[30] N. O. Umesi, J. T. Ideria and S. Abah, "Background Concentration of $\mathrm{NO}_{2}$ Prior to Construction and Operation of a Gas-Fired Gas Plant in the Niger Delta Region of Nigeria," Environment and Ecology, Vol. 27, No. 2, 2009, pp. 592-602.

[31] L. Rantanen, S. Mikkonen, L. Nylund, P. Kociba, M. Lappi and N. O. Nylund, "Effect of Fuel on the Regulated, Unregulated and Mutagenic Emissions of DI Diesel Engine," SAE Technical Paper No. 932686, Society of Automotive Engineers, Warrendale, 1993.

[32] J. Bunger, J. Krahl, K. Baum, O. Schroder, M. Muller, G. Westphal, P. Ruhnau, T. Schulz and E. Hallier, "Cytotoxic and Mutagenic Effects Particles Size and Concentration Analysis of Diesel Engine Emissions Using Biodiesel and Petro Diesel as Fuel," Archieves of Toxicology, 
Vol. 74, No. 8, 2000, pp. 490-494. doi: $10.1007 / \mathrm{s} 002040000155$

[33] Y. C. Lin, W. J. Lee and G. C. Fang, "Characterization of Particles Size Distribution from Diesel Engines Fueled with Palm-Biodiesel Blends and Paraffinic Fuel Blends," Atmospheric Environment, Vol. 42, 2008, pp. 1133-1143.

[34] J.-H. Tsai, K.-L. Huang, C.-H. Chiu, C.-C. Lin, W.-C. Kuo, W.-Y. Lin, H.-C. Chaung, T.-H. Yang and S.-J. Chen, "Particle-Bound PAHs and Particle-Extract-Induced $\mathrm{Cy}-$ totoxicity of Emission from a Diesel-Generator Fuelled with Soy-Biodiesel," Aerosol and Air Quality Research, Vol. 11, 2011, pp. 822-836.

[35] J. Yanowitz, R. L. McCormick and M. S. Graboski "InUse Emission from Heavy-Duty Diesel Vehicles," Environmental Science \& Technology, Vol. 34, No. 5, 2010, pp. 729-740. doi:10.1021/es990903w

[36] S. I. Efe, "Spatial Distribution of Particulate Air Pollution in Nigerian Cities: Implications for Human Health," Journal of Environmental Health Research, Vol. 7, No. 2, 2008, pp. 107-116.

[37] W. J. Lee, Y. F. Wang, T. C. Lin, Y. Y. Chen, W. C. Lin, C. C. Ku and J. T. Cheng, "PAH Characteristics in the Ambient Air of Traffic-Source," Science of the Total Environment, Vol. 195, No. 2-3, 1995, 185-200. doi:10.1016/0048-9697(95)04323-S

[38] R. M. Harrison, D. J. T. Smith and L. Luhana, "Source Apportionment of Atmospheric Polynuclear Aromatic Hydrocarbons Collected from an Urban Location in Birmingham, UK," Environmental Science and Technology, Vol. 30, No. 3, 1996, pp. 825-832. doi:10.1021/es950252d

[39] Y. C. Lin, W. J. Lee, C. C. Chen and C. B. Chen, "Saving Energy and Reducing Emissions of Both Polycyclic Aromatic Hydrocarbon and Particular Matter by Adding Bio-Solution to Emulsified Diesel," Environmental Science and Technology, Vol. 40, No. 17, 2006, pp. 55435559. doi:10.1021/es061120v

[40] Y. C. Lin, W. J. Lee and C. B. Chen, "Characterization of Polycyclic Aromatic Hydrocarbon from Diesel Engine by Adding Light Cycle Oil to Premium Fuel," Journal of the Air and Waste Management Association, Vol. 56, No. 6, 2006, pp. 725-758. doi:10.1080/10473289.2006.10464494

[41] Y. C. Lin, W. J. Lee, C. B. Chen, G. C. Fang and P. J. Tasi, "Impact of Using Fishing Boat Fuel with Higher Polyaromatic Content on the Emission of Polycyclic Aromatic Hydrocarbons from the Diesel Engine," Atmospheric Environment, Vol. 40, No. 9, 2006, pp. 16011609. doi:10.1016/j.atmosenv.2005.11.013

[42] Y. C. Lin, W. J. Lee and H. C. Hou, "PAH Emissions and Energy Efficiency of Palm-Biodiesel Blends Fueled on Diesel Generator," Atmospheric Environment, Vol. 40, No. 21, 2006, pp. 3930-3940. doi:10.1016/j.atmosenv.2006.02.026

[43] Y. C. Lin, W. J. Lee, T. S. Wu and C. T. Wang, "Comparison of PAH and Regulated Harmful Matter Emissions from Biodiesel Blends and Paraffin Fuel Blends on Engine Accumulated Mileage Test," Fuel, Vol. 85, No. 17-18, 2006, pp. 2516-2523. doi:10.1016/j.fuel.2006.04.023

[44] H. H. Yang, C. F. Chiang, W. J. Lee, K. P. Hwang and M. F. Wu, "Size Distribution and Dry Deposition of Road Dust PAHs," Environment International, Vol. 25, No. 5, 1999, pp. 585-597. doi:10.1016/S0160-4120(99)00036-7

[45] G. R. Ana, "Air Pollution in the Niger Delta Area: Scope, Challenges and Remedies," In: M. Khallaf, Ed., The Impact of Air Pollution on Health, Economy, Environment and Agricultural Sources, InTech-Open Access Company, Rijeka, 2011. pp. 182-198.

[46] L. S. Kalkstein, "A New Approach to Evaluate the Impact of Climate on Human Mortality," Environmental Health Perspectives, Vol. 96, 1991, pp. 145-150. doi:10.1289/ehp.9196145

[47] M. Saez, J. Sunyer, A. Tobias, F. Ballester and A. M. Antó, "Ischaemic Heart Disease and Weather Temperature in Barcelona, Spain," European Journal of Public Health, Vol. 10, No. 1, 2000, pp. 58-63. doi:10.1093/eurpub/10.1.58

[48] L. Makra, S. Z. Tombácz, B. Bálint, Z. Sümeghy, T. Sánta and T. Hirsch, "Influences of Meteorological Parameters and Biological Chemical Air Pollutants on the Incidence of Asthma and Rhinitis," Climate Research, Vol. 37, No. 1, 2008, pp. 99-119. doi:10.3354/cr00752

[49] Z. Sümeghy, "Influence of Different Factors on Relative air Humidity in Szeged, Hungary," Acta Climatologica et Chorologica, Vol. 42-43, 2009, pp. 131-141.

[50] P. Wolkoff and S. K. Kjærgaard, "The Dichotomy of Relative Humidity on Indoor Air Quality," Environment International, Vol. 33, No. 6, 2007, pp. 850-857. doi:10.1016/j.envint.2007.04.004

[51] N. L. Nagda and H. E. Rector, "A Critical Review of Reported Air Concentrations of Organic Compounds in Aircraft Cabins," Indoor Air, Vol. 13, No. 3, 2003, pp. 292301. doi:10.1034/j.1600-0668.2003.00202.x

[52] S. Yusoff, "Renewable Energy from Palm Oil-Innovation on Effective Utilization of Waste," Journal of Cleaner Production, Vol. 14, No. 1, 2006, pp. 87-93. doi:10.1016/j.jclepro.2004.07.005

[53] K. W. Chan, I. Watson and K. C. Lim, "Use of Palm Oil Waste Material for Increased Production," In: E. Pusshparajah and S. L. Anu, Eds., Soil Science and Agricultural Development in Malaysia, Malaysia Society of Soil Science, Kuala Lumpur, 1980, pp. 213-242.

[54] O. Taiwo, "The Case of Lagos-Air Quality Improvement Project," Lagos Metropolitan Area Transport Authority (LAMATA), 2005.

[55] FEPA, "Guidelines and Standards for Environmental Pollution Control in Nigeria," Federal Environmental Government Protection Agency, 1991.

[56] S. C. A. de Almeida, C. R. Belchior, M. V. G. Nascimento, L. S. R. Vieira and G. Fleury, "Performance of a Diesel Generator Fuelled with Palm Oil," Fuel, Vol. 81, No. 6, 2002, pp. 2097-2102. doi:10.1016/S0016-2361(02)00155-2

[57] M. S. Graboski and R. L. McCornimik, "Combustion of Fat and Vegetable Oil Derived Fuels in Diesel Engines," Progress in Energy and Combustion Science, Vol. 24, No. 
2, 1998, pp. $125-164$. doi:10.1016/S0360-1285(97)00034-8

[58] Committee of the Environmental and Occupational Health Assembly of the American Thoracic Society, "Health Effects of Outdoor Air Pollution [Review]," American Journal of Respiratory and Critical Care Medicine, Vol. 153, No. 1, 1996, pp. 3-50.

[59] P. Ahlink, L. Ntziachristos, J. Keskinen and A. Virtanen, "Real-Time Measurements of Diesel Particle Size Distribution with an Electrical Low-Pressure Impactor," SAE Technical Paper No. 980410, 1998.

[60] Z. Ning, C. S. Cheung and S. X. Liu, "Experimental Investigation of the Effect of Exhaust Gas Cooling on Diesel Particulate," Journal of Aerosol Science, Vol. 35, No. 3, 2004, pp. 333-345. doi:10.1016/j.jaerosci.2003.10.001

[61] E. K. Ossai, G. O. Iniaghe, S. A. Osakwe and P. A. Agbaire, "Pollution Problems and Environmental Effects of Chemicals," In: F. K. Ekechi, Reading in General Studies: History and Philosophy of Science, Abraka General Studies Department Publishers DELSU, Abraka, 1999, pp. 83-86.

[62] J. J. McAughey, "Regional Lung Deposition and Dose of Ambient Particulates in Humans by Particle Mass and Number," Research Report, AEA Technology, Aerosol Science Centre, Oxfordshire, 1997.

[63] J. Injuk and R. Van Gricken, "Atmospheric Concentrations and Deposition of Heavy Metals over the North Sea: a Literature Review," Journal of Atmospheric Chemistry, Vol. 20, No. 2, 1995, pp. 179-212.
doi:10.1007/BF00696557

[64] C. A. III. Pope, D. V. Bates and M. E. Raizenne, "Health Effect of Particulate Air Pollution, Time for Reassessment," Environmental Health Perspectives, Vol. 103, No. 5, 1995, pp. 472-480. doi:10.1289/ehp.95103472

[65] D. R. Blake and F. S. Rowland, "Urban Leakage of Liquefied Petroleum Gas and Its Impact on Mexico City Air Quality," Science, Vol. 269, No. 5226, 1995, pp. 953-956. doi:10.1126/science.269.5226.953

[66] P. Wolkoff, "Impact of Air Velocity, Temperature, Humidity, and Air on Long-Term VOC Emissions from Building Products," Atmospheric Environment, Vol. 32, No. 14-15, 1998, pp. 2659-2668. doi:10.1016/S1352-2310(97)00402-0

[67] L. Fang, G. Clausen and P. O. Fanger, "Impact of Temperature and Humidity on Chemical and Sensory Emissions from Building Materials," Indoor Air, Vol. 9, No. 3, 1999, pp. 193-201. doi:10.1111/j.1600-0668.1999.t01-1-00006.x

[68] A. Litvak, A. J. Gadgil and W. J. Fisk, "Hygroscopic Fine Mode Particle Deposition on Electronic Circuits and Resulting Degradation of Circuit Performance: An Experimental Study," Indoor Air, Vol. 10, No. 1, 2000, pp. 47 56. doi:10.1034/j.1600-0668.2000.010001047.x

[69] A. F. Miguel, A. H. Reis and M. Aydin, "Aerosol Particle Deposition and Distribution in Bifurcating Ventilation Ducts," Journal of Hazardous Material, Vol. 116, No. 3, 2004, pp. 249-255. doi:10.1016/j.jhazmat.2004.09.013 